\title{
NEUROPSYCHOPHARMACOLOGY REVIEWS What has sex got to do with it? The role of hormones in the transgender brain
}

\author{
Hillary B. Nguyen ${ }^{1,2,3}$, James Loughead ${ }^{3}$, Emily Lipner ${ }^{1,2}$, Liisa Hantsoo ${ }^{3}$, Sara L. Kornfield ${ }^{3}$ and C. Neill Epperson ${ }^{1,2,3,4}$
}

\begin{abstract}
Sex differences and hormonal effects in presumed cisgender individuals have been well-studied and support the concept of a mosaic of both male and female "characteristics" in any given brain. Gonadal steroid increases and fluctuations during peri-puberty and across the reproductive lifespan influence the brain structure and function programmed by testosterone and estradiol exposures in utero. While it is becoming increasingly common for transgender and gender non-binary individuals to block their transition to puberty and/or use gender-affirming hormone therapy (GAHT) to obtain their desired gender phenotype, little is known about the impact of these manipulations on brain structure and function. Using sex differences and the effects of reproductive hormones in cisgender individuals as the backdrop, we summarize here the existing nascent neuroimaging and behavioral literature focusing on potential brain and cognitive differences in transgender individuals at baseline and after GAHT. Research in this area has the potential to inform our understanding of the developmental origins of gender identity and sex difference in response to gonadal steroid manipulations, but care is needed in our research questions and methods to not further stigmatize sex and gender minorities.
\end{abstract}

Neuropsychopharmacology (2019) 44:22-37; https://doi.org/10.1038/s41386-018-0140-7

\section{INTRODUCTION}

\section{Definitions}

The term gender identity refers to a person's innermost sense of their own gender, while sex is the sex assigned at birth by a physician, typically based on chromosomal, hormonal, physical, or anatomical characteristics [1] (Table 1). For clarity in this review, sex will be used interchangeably with natal sex, biological sex, and sex assigned at birth. Most people identify as cisgender (abbreviated cis), a term which describes an alignment of gender identity with natal sex. For transgender people (abbreviated trans), gender identity, expression (i.e., the gender one presents to others as), and/or behavior (i.e., how the person chooses to express their gender identity through actions) are different from that typically associated with their natal sex, as defined by the norms of a given culture [1-3]. Studies to date have focused primarily on the two binary populations of transgender individuals: male-to-female (MTF) and female-to-male (FTM). MTF individuals, also referred to as transgender females, were assigned male at birth but identify as female. FTM individuals, also referred to as transgender males, were assigned female at birth but identify as male. Prevalence rates of gender dysphoria (GD) and the number of transgender-identifying individuals seeking help with transition have been rising worldwide over the past two decades [4]. In 2016, the Williams Institute estimated that $\sim 0.6 \%$ of US adults identify as transgender when considering a binary definition [5]. Previous studies of transgender populations have not considered individuals across the gender spectrum, including those who identify as gender non-binary, gender fluid, transmasculine, or transfeminine. Transmasculine is an umbrella term that includes FTMs and is used to describe people who were assigned female at birth but identify with masculinity to a greater extent than femininity, while transfeminine is an umbrella term that includes MTFs and describes those who were assigned male at birth but identify with femininity to a greater extent than masculinity [6].

Previous attempts to codify the transgender experience labeled it as a pathological condition called Gender Identity Disorder (GID) in the American Psychiatric Association's (APA) Diagnostic and Statistical Manual of Mental Disorders (DSM) Third and Fourth Editions $[7,8]$. Thus, transgender individuals automatically carried a "psychiatric" disorder. In contrast, DSM-5 criteria for GD is given to transgender individuals only if they feel significant distress or impairment in functioning as a result of experiencing a "strong and persistent incongruence between their gender identity and assigned sex at birth" [9]. There is an ongoing debate about whether this newer definition of GD should even be included in the DSM given the continued potential stigma of characterizing the difficulties transgender individuals experience as an illness in our predominantly gender-binary society. However, the diagnosis of GD can sometimes be necessary to receive services covered by health insurers [10]. Epidemiologic data about the prevalence of GD are unreliable, especially considering the sociocultural variations among different countries and societies, but in 2013 the DSM-5 offered conservative approximations of $0.005-0.014 \%$ for biological males and $0.002-0.003 \%$ for biological females [9]. These estimates are considerably lower than the aforementioned statistic for transgender identification reported by the Williams Institute because individuals may identify as transgender without experiencing gender dysphoric symptoms or receiving the diagnosis of GD.

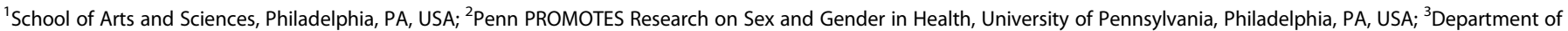
Psychiatry, Philadelphia, PA, USA and ${ }^{4}$ Department of Obstetrics and Gynecology, Perelman School of Medicine at the University of Pennsylvania, Philadelphia, PA, USA
} Correspondence: C. Neill Epperson (cepp@pennmedicine.upenn.edu) 
Table 1. Gender terminology

\begin{tabular}{|ll}
$\begin{array}{l}\text { Term } \\
\text { Gender identity }\end{array}$ & $\begin{array}{l}\text { Definition } \\
\text { Sex }\end{array}$ \\
& $\begin{array}{l}\text { A person's innermost sense of their own gender (e.g., male, female, a blend of both, neither). } \\
\text { hormonal, physical, or anatomical characteristics of the person. In this article, sex is used synonymously with sex } \\
\text { assigned at birth, natal sex, and biological sex. } \\
\text { *Note: Many transgender people feel stigmatized by the terms natal sex and biological sex because they are often } \\
\text { used to invalidate their gender identities. }\end{array}$ \\
Cisgender (abbreviated cis) & A term used to describe a person whose gender identity aligns with their sex assigned at birth. \\
Transgender (abbreviated trans) & $\begin{array}{l}\text { A term used to describe a person whose gender identity, expression (i.e., the gender one presents to others as), and/ } \\
\text { or behavior (i.e., how the person chooses to express their gender identity through actions) is different from cultural } \\
\text { expectations typically associated with the sex they were assigned at birth. } \\
\text { Used to describe a transgender person assigned male at birth who identifies as female. } \\
\text { Male-to-female (MTF) } \\
\text { In this article, the term MTF transgender individual is used synonymously with transgender female and transgender } \\
\text { woman. } \\
\text { Used to describe a transgender person assigned female at birth who identifies as male. In this article, the term FTM } \\
\text { transgender individual is used synonymously with transgender male or transgender man. }\end{array}$
\end{tabular}

Early research seeking to determine the underlying causes of $\mathrm{GD}$ and the transgender identity focused almost primarily on sex differences and whether the brain of individuals with GD are more like their natal sex or that of their preferred gender. While we review the brain imaging studies related to this focus, we propose that this line of research can further stigmatize this population and does not address basic questions about the long-term safety of gender-affirming hormone therapies (GAHT) with respect to brain health. While low-quality evidence based on 247 transgender adults from three uncontrolled prospective cohort studies suggests that hormone therapy may lead to improvements in psychological functioning [11-13], there are no major longitudinal studies of GAHT use beyond 18 months with respect to brain structure, function, or cognition among adolescent or adult transgender individuals. Reproductive hormones have profound and broad impact on neurochemistry, neuronal structure, glial architecture, and regional brain function during development as well as adulthood [14, 15]. Moreover, loss of estradiol [16-18] and declines in testosterone $[19,20]$ can result in cognitive and mood difficulties among adult cisgender women and men, respectively, suggesting that research focusing on the long-term safety of GAHT with respect to the central nervous system (CNS) is needed.

The purpose of this review is to summarize the literature on sex differences from the novel perspective of gender identity, to address deficits in our knowledge surrounding the transgender and gender non-binary patient population, and emphasize the importance of considering the long-term effects of GAHT on brain health, particularly the mental skills comprising executive function that enable us to accomplish daily tasks. Based on the literature in cisgender populations, which is summarized below, we believe that GAHT presents some unique concerns for executive functioning and cognitive control in transgender individuals. We will then present data on both "baseline" differences in MTF and FTM individuals before they have taken any GAHT, as well as how their CNS responds to GAHT. More attention and research dedicated to studying this growing population will ensure that all people receive optimal care regardless of gender identity. In fact, the Sexual and Gender Minority Research Office (SGMRO) of the National Institutes of Health (NIH) was officially established in 2015 to address health and research disparities in the LGBTQ + (lesbian, gay, bisexual, transgender, queer) populations. Investigation of risk for long-term executive functioning difficulties related to GAHT use in the transgender community would be conducive to the SGMRO mission.
The brain as a gender mosaic

To understand the status of neuroimaging research in transgender samples, it is important to appreciate the origins of sex differences in the brain across development in cisgender individuals. The presence of the androgen testosterone and its aromatization to estradiol in utero cause the fetal brain of the male to organize in such a manner to result in a more masculine phenotype across the lifespan $[15,21]$. The absence of androgen production by the female fetus and estrogen-binding activity of alpha-fetoprotein in the female fetus lead to a more feminine brain phenotype [22]. However, the distinction between the female and male brain is not always clear-cut, as brain regions may be more or less programmed by androgens and estrogens leading to greater or lesser masculinization of various brain regions during prenatal development. This "mosaic" of maleness and femaleness occurs even among cisgender individuals [23].

One compelling hypothesis for the development of transgender experiences is a disconnect between an individual's brain "sex/ gender" and the sex associated with their reproductive organs due to the relative maturation of these organs across gestation, with genital development completed in first trimester and brain development continuing throughout pregnancy [24, 25]. A genetic component may also be involved, as siblings of transgender individuals are more likely to be transgender compared with the general population [26].

At puberty, gonadal steroids act upon the programmed brain and, together with life experience and genetics, create a phenotype that is along the gender continuum [2, 16, 27]. Older studies of presumed cisgender individuals in the adolescent period noted that the structure of the prefrontal cortex undergoes significant changes during puberty and adolescence, with important physical and psychological variations between males and females [14, 28-30]. More recently, a study of a large cohort of children, adolescents, and young adults $(n=674)$ demonstrated that sex differences in patterns of cognition (spatial, motor, language, nonverbal reasoning, and emotion identification) are related to sex differences in patterns of resting-state functional connectivity, with males demonstrating more between-network connectivity, while similarly aged females displayed more withinnetwork connectivity [31]. Whether this is a true sex difference or due to variations in pubertal status between similarly aged females and males is not clear, as within and between network connectivity typically increases and decreases, respectively, across development [32, 33]. In another cohort of 1189 youth, data demonstrated mean brain gray matter density increased from 
childhood to young adulthood, while total brain gray matter volume and mean cortical thickness decreased with age [34]. Total gray matter mass only shows a slight decline. There is a male advantage in gray matter volume, though females have higher gray matter density throughout the brain. Two regions in which sex differences are most commonly reported are the medial temporal lobe structures of the hippocampus and amygdala [35, 36]. MRI studies have also demonstrated changes during adolescence to the frontal lobes, a region strongly associated with executive function [37]. Executive function is a major branch of cognition, including sustained attention, cognitive control, inhibition, error monitoring, and working memory [33]. One possible explanation for these structural differences in the frontal lobes is the regionally specific actions of sex steroids during pubertal maturation [27], particularly in the dorsolateral prefrontal cortex [38]. It follows that puberty blocking with gonadotropin releasing hormone $(\mathrm{GnRH})$ agonist injections or implants, and/or exogenous hormone administration would have important implications for executive function development during the transition from childhood to young adulthood. At the moment, this area of research remains largely unexplored in adolescents and emerging adults and therefore warrants further investigation.

By adulthood, absolute brain and corpus callosum volume is larger in males than in females, even after accounting for body size $[39,40]$. A recent meta-analysis of MRI studies confirms that, on average, absolute intracranial volume, total brain volume, cerebrum, gray matter, white matter, cerebrospinal fluid volume, and cerebellum are greater in males than females [41]. Men also have a higher proportion of white matter, though women have a higher proportion of gray matter [40,42]. The volume of the central subdivision of the bed nucleus stria terminalis (BNSTc), a brain region implicated in anxiety and stress regulation [43], has been shown to be larger in men than women [44, 45]. On the other hand, females tend to have thicker cortices than males as well as higher global cerebral blood flow [42], perhaps secondary to the vasodilatory effects of estradiol [46]. Sex differences among reproductive-aged, cisgender adults have also been consistently reported in functional connectivity during a resting state [47-51].

With respect to cognitive function, verbal and spatial tasks show a female and male sex bias, respectively. Adult women are also reported to outperform men on tasks involving perceptual speed

Male

In utero
- Testosterone and its
aromatization to estrogen
cause masculinization of
the fetal brain
Adolescence
- More between-network
connectivity
- Larger grey matter volume
- Lower grey matter density
Adulthood
- More total brain volume
- More grey matter volume
- More white matter volume
- More cerebrospinal fluid volume
- Higher proportion of white matter
- Larger volume of the central
subdivision of the bed nucleus
stria terminalis
- Better visuospatial and
mathematical ability
- Weaker right-hand preference

Fig. 1 Sex differences in brain structure and function across the lifespan among presumed cisgender individuals and fine manual dexterity, whereas visuospatial and mathematical abilities are typically greater in males $[52,53]$. In general, males tend to process verbal stimuli in a more lateralized way and show a weaker right-hand preference than females [54, 55].

Figure 1 summarizes the relative sex differences in brain structure and function across development among presumed cisgender individuals. Though few asked participants about their gender identity, these studies regarding sex differences have motivated an entire area of research that aims to understand the etiology of being transgender. Causal mechanisms are unknown, though brain imaging methods are being applied with some promising, if inconsistent, results.

\section{Reproductive hormones and the cisgender brain}

Overall, estradiol, testosterone, and progesterone, as well as its neurosteroid metabolite allopregnanolone (Allo) have great influence on neurotransmitter levels and receptors, and neuronal and glial architecture and function in presumably cisgender individuals, as described above. Thus, fluctuations in these gonadal and adrenal steroids can produce profound effects on brain and mental health.

The effect of a natural or premature menopause on the brain and behavior of cisgender females has been well-studied and could theoretically inform our understanding of the long-term impact of GAHT in trans males. While the cis-gender postmenopausal woman is hypogonadal with respect to sex hormones and neuroactive steroids, the trans-male using GAHT will have low levels of estradiol, progesterone and Allo, but levels of testosterone that are typically supraphysiologic for individuals with two $\mathrm{X}$ chromosomes. While transitioning to menopause, many presumably cis-gender women report a decline in cognitive function [56], particularly in memory and attention $[17,18,57]$ and increase in risk for major depressive disorder [58]. Moreover, functional magnetic resonance imaging (fMRI) studies in humans highlight the importance of estradiol for intact prefrontal executive network function and connectivity among menopausal women [16, 59-61]. Longitudinal studies reveal that women who experience premature hormonal menopause and estrogen deficiency before reaching the median age of natural menopause are at heightened risk for first onset depression, as well as morbidity (e.g., cardiovascular disease, neurological diseases, psychiatric diseases) and mortality [62]. However, some of these adverse outcomes may be prevented by exogenous estrogen treatment initiated proximal to the final menstrual period and continued into the postmenopause [63].

Conversely, androgens have varying impact on brain and behavior across the female lifespan and have been well-studied in cis-gender males and females [64]. Increased levels of androgens have been linked to increases in irritability, hostility, and clinical mood disorders in women [64, 65]. For example, higher free testosterone levels have been associated with premenstrual syndrome and depression in normally cycling women, with anger and depression among pregnant and postpartum women and with increased hostility in post-menopausal women [64].

Polycystic ovary syndrome (PCOS) is a natural model for the study of androgen excess among cisgender females. PCOS affects almost $15 \%$ of women of reproductive age [66] and is characterized by chronically high free testosterone levels and low levels of progesterone and estradiol from puberty onward [64]. Females with PCOS are at substantial risk for psychological, metabolic, reproductive, and cardiovascular abnormalities $[67,68]$, such as acne, obesity, type 2 diabetes, heart disease, and mood disorders like depressive, bipolar, and anxiety disorders, as well as lowered health-quality of life [65, 69-74].

On the contrary, low testosterone in cisgender men is linked to numerous physical and mental health problems. In a populationbased cohort of cisgender men, serum testosterone levels are inversely related to mortality due to cardiovascular disease and cancer [75]. The presence of low testosterone also predicts the 
development of metabolic syndrome, type 2 diabetes, frailty, and depressive symptoms in males [76-78]. In an observational cohort of middle-aged cisgender men $(n=398)$ with low total testosterone levels and high chronic medical morbidity, testosterone treatment was associated with decreased mortality compared with no testosterone treatment [79].

In combination, these findings that indicate the physical and mental health ramifications of fluctuations in reproductive hormones in the cisgender population warrant further investigation into the long-term safety of GAHT for transgender individuals. Of particular interest is high-dose testosterone that suppresses estradiol levels in FTMs due to the potential long-term effects of low levels of estradiol and supra-physiologic levels of testosterone.

Transgender sex differences prior to GAHT

Although sex differences in presumably cisgender individuals have been well-studied, little is known about how gender identity intersects with these sex differences in brain function and structure among people who identify as transgender, particularly prior to GAHT. Early brain studies on the transgender population compared the brain structures of MTFs and FTMs prior to GAHT with both cisgender male and cisgender female controls to identify whether there are existing brain differences in MTF and FTM individuals at baseline (before hormone treatment) that set them apart from cisgender individuals. Most studies focused on MTFs, resulting in limited information about FTMs. Additionally, the majority of these studies were cross-sectional and limited to the above brain regions already showing sex differences among cisgender populations, while neglecting structures underlying cognitive and emotion processing tasks required for optimal dayto-day functioning.

Recent application of neuroimaging techniques has advanced our understanding of baseline differences between brain regions as well as the impact of GAHT on performance in cognitive tasks that exhibit sex-biased responses. These studies only examined the sex-biased verbal and spatial tasks, while overlooking other cognitive domains. To the best of our knowledge, studies thus far have not systematically considered executive functions such as sustained attention and focus, working memory, impulse control, affective regulation, and motivation and activation for work.

Presently, findings are conflicting about whether the neuroanatomy of transgender individuals prior to GAHT resembles that of their biological sex or their gender identity, but most crosssectional neuroimaging research indicates that brain morphology and activation patterns at rest and during cognitive performance are more congruent with gender identity than natal sex in untreated MTFs and FTMs. Among transgender individuals meeting criteria for GD, cortical thickness $[80,81]$, gray matter volume $[80,82]$, white matter microstructure [83, 84], structural connectivity [85], and corpus callosum shape [86] have been found to be more similar to cisgender control subjects of the same preferred gender compared with those of the same natal sex. Likewise, transgender individuals feature a unique pattern of connections in the resting state functional connectivity network that differ from those of their natal sex [87].

Performance on cognitive tasks by MTFs and FTMs prior to GAHT is often more congruent with gender identity. In most cases, investigators have focused on verbal and spatial tasks which display a clear sex bias. Verbal tasks show a female sex bias, while spatial tasks show a male sex bias. Multiple studies conclude that MTFs perform significantly better on female-favoring verbal tests compared to cisgender male controls [88-90], whereas FTMs perform significantly worse than cisgender female controls on verbal tests [90]. Measuring conceptual styles, MTFs and FTMs perform in congruence with their gender identity [91]. This suggests that the tendency towards "feminization" in MTFs and "masculinization" in FTMs is an innate quality unrelated to exogenous hormonal intervention, and more closely tied to brain development in transgender individuals.

Functional neuroimaging also confirms that activation patterns in FTMs and MTFs before GAHT intervention are more representative of their gender identity than natal sex. MTFs exhibit a pattern of hypothalamic activation consistent with their preferred gender when smelling odorous steroids 4,16androstadien-3-one and estra-1,3,5(10),16-tetraen-3ol, occupying an intermediate position with predominantly female-like features [92]. Furthermore, cerebral activation pattern in MTFs are similar to that of cisgender female controls during viewing of erotic stimuli, indicating female-like cerebral processing in MTFs [93]. When evaluating voice gender perception of male and female voices in MTFs compared with cisgender men and cisgender women, neural activation in the superior frontal gyrus in MTFs was more similar to that of cisgender women than cisgender men [94].

Nevertheless, approximately one-quarter of the literature has presented conflicting data. More specifically, these cross-sectional in vivo studies suggest the opposite - that brain morphology and cognitive performance show greater congruence with natal sex than gender identity in untreated MTFs and FTMs. In the context of morphology, gray matter distribution and regional volumes in MTF and FTM adolescents are in accordance with their respective natal sex [95]. MTFs have regional gray matter variation more similar to that of healthy male controls (natal sex) than to female controls (gender identity) [96]. However, MTFs show significantly larger volumes of regional gray matter in the putamen compared with the male controls, indicating that the putamen is more feminized (cisgender females typically have the largest gray matter volumes out of all groups). Likewise, FTMs share a similar pattern with heterosexual cisgender female controls concerning cortical thickness, surface area, gray matter volume, structural volumes (amygdala, hippocampus, caudate), and resting state functional connectivity from the amygdala [97]. Performance has also been found to align more with natal sex than gender identity, according to the Wechsler Adult Intelligence Scale (WAIS) [91] and when testing four potentially sex-sensitive cognitive factors (rotation, visualization, perception, and verbalization) [98]. While the majority of the literature indicates that brain structure and function are more in line with gender identity than natal sex, even in trans individuals who have not undergone GAHT, the equivocal nature of some findings suggests that perhaps we ought to consider a continuum of gender identity where individuals may not fit neatly into binary characterizations. Furthermore, it is important to consider that many studies were undertaken with small sample sizes and results should be interpreted cautiously. Given these contradictory findings, replication of this research in larger samples is needed.

\section{Gender-affirming hormone therapy}

Over the past few years, it has become increasingly common and accepted for transgender people to socially transition to their gender identity. This includes both medical and non-medical steps. Some choose to present as their gender identity by changing their appearance (e.g., hair, clothing), pronouns, and/or name. Other transgender individuals decide to seek medical treatments, such as GAHT and surgery (e.g., breast augmentation, phalloplasty), to achieve the desired physical anatomy of their gender identity. An early intervention for children who express gender non-conformity or GD during the pre- and early-pubertal years includes blocking pubertal development. Puberty blockers are a reversible strategy to delay puberty and give patients and families time to develop a transition plan [99]. After puberty, GAHT can be taken to reduce endogenous hormone levels of the biological sex and to replace them with those of the desired gender [100]. MTFs typically take estradiol and androgen blockers 
Table 2. GAHT effects on brain structure (Structural MRI)

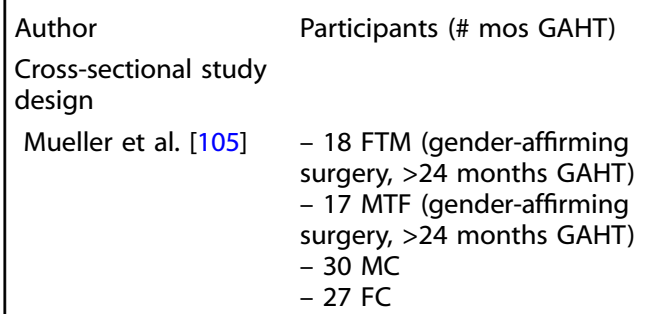

Spizzirri et al. [106] - 20 MTF (GAHT naïve)

- 20 MTF (>12 months GAHT)

$-20 \mathrm{MC}$

$-20 \mathrm{FC}$

Longitudinal study

design

Hulshoff Pol et al.

[111]

- 6 FTM (4 months GAHT)

- 9 MC (1 year between scans)

- 6 FC (1 year between scans)

1.5 Tesla magnet structural/volumetrics

3 Tesla magnet structural/volumetrics

1.5 Tesla magnet structural/volumetrics

3 Tesla magnet diffusion tensor imaging

3 Tesla magnet structural/volumetrics

Zubiaurre-Elorza et al. - 14 MTF (6 months)

[109]

$$
\text { - } 15 \text { FTM (6 months) }
$$

Seiger et al. [108]

- 25 FTM (>4 months GAHT)

- 14 MTF (>4 months GAHT)

- 14 FC (2-4 months between scans)

- 12 MC (1-4 months between scans)

Burke et al. [110]

$$
\begin{aligned}
& -22 \text { FTM ( } 14.4 \pm 6 \text { months } \\
& \text { GAHT) } \\
& -12 \text { FC ( } 34.8 \pm 6 \text { months } \\
& \text { between scans) } \\
& -7 \text { MC ( } 34.8 \pm 6 \text { months) } \\
& \text { between scans }
\end{aligned}
$$

3 Tesla magnet structural/volumetrics

\section{Tesla magnet} structural/volumetrics, diffusion imaging, functional connectivity

3 Tesla magnet diffusion-weighted imaging

\author{
- 25 FTM (baseline, 1 month \\ and 4 months after GAHT) \\ -12 FC \\ $-13 \mathrm{MC}$
}

Results

- The mean neuroanatomical volume for the amygdala, putamen, and corpus callosum differed between MTFs and FCs, but not between MTFs and MCs.

- Several brain structures, including the medial temporal lobe structures and cerebellum, were found to be different in volume between FTMs and MCs.

- FTMs and FCs had volume differences in the medial temporal lobe, nucleus accumbens, and $3^{\text {rd }}$ ventricle.

- MCs had larger cerebellar volumes and a smaller anterior corpus callosum than FCs.

- Compared with MCs and treated MTFs, FCs had lower global brain volumes and regional gray matter volumes in a large portion of the posterior-superior frontal cortex.

- Both naïve MTFs and treated MTFs had lower bilateral insular gray matter volumes than FCs.

- Four months of anti-androgen and estrogen GAHT in MTFs decreased hypothalamic and total brain volumes and increased ventricle volume "toward female proportions" compared with MCs over one year.

- Four months of androgen GAHT in FTMs increased total brain and hypothalamic volumes and decreased ventricle volume "toward male proportions" compared with FCs over one year.

- Prior to GAHT, the white matter microstructure in FTMs differs from that of FCs but not MCs.

- At least 7 months of androgen GAHT changes white matter microstructure in FTMs, as measured by fractional anisotropy (FA) values. After 7 months of GAHT, FA increased in the right superior longitudinal fasciculus (SLF) and right corticospinal tract (CST) compared with pre-GAHT values.

- These increments in FA in the SLF and CST were predicted by the free testosterone index before GAHT, but not after GAHT.

- Six months of estrogen GAHT in MTFs decreased cortical thickness and subcortical volumetric measures, and increased ventricle volume.

- Six months of testosterone GAHT in FTMs increased cortical thickness.

- A positive correlation between serum testosterone and free testosterone index changes and cortical thickness changes was found in the parieto-temporo-occipital regions of FTMs.

- MTFs receiving estradiol and anti-androgens for at least 4 months had gray matter decreases in the right hippocampal region. These decreases were reflected by increases in the third and lateral ventricles.

- Changes in gray matter structures in MTFs were correlated with changes in progesterone levels.

- Prior to GAHT, the medial prefrontal cortex (mPFC) was thicker in FTMs than in both FCs and MCs, and remained unchanged after GAHT.

- Compared with the FCs and MCs, testosterone GAHT in FTMs resulted in increased bilateral cortical thickness in the insular and superior cortices, changes in cortico-cortical thickness covariation between mPFC and occipital cortex, increased fractional anisotropy in the posterior portion of the frontooccipital tract connecting these regions, and increased functional connectivity between mPFC and temporo-parietal junction.

- FTMs showed testosterone-related reductions in mean diffusivity in the lateral hypothalamus. Significant reductions were shown unilaterally after 1 month and bilaterally after 4 months of testosterone GAHT.

- The magnitude of reductions in mean diffusivity were significantly associated with GAHT-induced increases in free androgen index and bioavailable testosterone.

GAHT gender-affirming hormone therapy, GDF gender dysphoric female, GDM gender dysphoric male, MTF male-to-female transgender, FTM female-to-male transgender, $M C$ cisgender male control, $F C$ cisgender female control 
to feminize their bodies, while FTMs typically take testosterone to masculinize their bodies. Examining the effects of GAHT on the brain during these neuroplastic periods is critical.

Numerous studies have focused on the effects of endogenous and exogenous hormones in cisgender individuals, but research about GAHT is in its early stages. The National Transgender Discrimination Survey Report on Health and Health Care finds that at least $80 \%$ of transgender people have either taken GAHT or want to take GAHT at some point [101]. The benefit of GAHT to behavioral health and quality of life acutely is considerable [102]. However, there is little information about the safety and efficacy of these therapies. Transgender and gender non-binary patients, their families, and their providers are concerned about whether clinically prescribed GAHT will affect cognitive domains that are important for everyday function, particularly if initiated during windows of development such as peri-adolescence to young adulthood when the executive network is under rapid development. Unfortunately, there is little information to guide clinical decision-making at this time. To be most helpful to this population, research should now embrace gender expansive identities (including those that do not fall within the gender binary) and focus on collecting data that have the potential to improve health over the entire lifespan, namely by conducting studies that evaluate long-term cognitive and behavioral effects of GAHT.

While sex hormones can exert an organizational influence on brain morphology during prenatal and peri-pubertal development, as discussed above, sex steroids can still affect the brain later in adulthood when the brain has fully developed, as in the case of GAHT. Most research on the effects of GAHT thus far has focused on metabolism and the vascular, epithelial, and musculoskeletal systems, while neglecting the central nervous system (CNS) [103]. For instance, testosterone GAHT causes transgender men to develop a different body composition with more muscle mass and strength, less fat mass, and an altered bone geometry with larger bones compared with female controls [104].

Only a few studies have prioritized brain health as a function of exogenous hormone administration, sex, and gender identity which is the focus of this review. Below is a summary of the existing literature in the English language on how brain imaging outcomes, performance on cognitive tasks, and mood within the adult transgender population are affected by GAHT. Note that transgender individuals who were not taking GAHT at the time of their respective studies were sometimes labeled as "gender dysphoric" by researchers, while those who were taking or had previously taken GAHT were considered "transgender."

\section{METHODS}

All studies relevant to the topic and written in English were included. Most research articles featured in this review were found using Google Scholar and PubMed. Publications cited by these articles were also referenced. Search terms included "transgender," "transsexual," "hormones," "brain," and "imaging." Only studies that directly focused on subjects who do not identify with their sex assigned at birth and looked at GAHT effects were included. Publications that did not look at brain structure, function, or behavior were not included. Furthermore, we did not use a cutoff date for the year of publication or a limit on country of study. Both cross-sectional and longitudinal studies were accepted, but data from longitudinal studies were given greater emphasis. Articles were excluded if it was not clear from the description whether participants were naïve to GAHT. Adolescents and children taking puberty blockers rather than cross-sex hormones were also not considered in this review.

\section{RESULTS}

GAHT effects on brain structure

Cross-sectional studies. One small cross-sectional study assessed the effects of at least 2 years of GAHT in 18 FTMs and 17 MTFs after gender-affirming surgery. Neuroanatomical differences in the brain were found to be region-specific between transgender individuals and their biological sex as well as their gender identity, suggesting localization of influence by sex hormones on brain structure [105]. More specifically, the mean neuroanatomical volume for the amygdala, putamen, and corpus callosum of MTFs was found to be significantly different from those of cisgender women, but not cisgender men (consistent with the natal sex of MTFs). FTMs, on the other hand, differed from cisgender women in the third ventricle and nucleus accumbens, which is consistent with their preferred gender, and differed from cisgender men in the medial temporal lobe structures and cerebellum. FTMs differed from both cismen and ciswomen in fusiform volume. These findings suggest that brain structures are not uniformly influenced by GAHT, and that while some structures become more like those of the preferred gender, others may not, or fall somewhere in the middle. This lends credence to the concept of the gender mosaic, which falls between the two rigidly defined binary gender identities.

A similar finding of region-specific volumetric differences between gender and natal sex was noted in a study with 20 GAHT-naïve MTFs, 20 MTFs treated with GAHT for at least 1 year, 20 cisgender men controls, and 20 cisgender women controls [106]. The cisgender women had lower global brain volumes and regional gray matter volumes in a large portion of the posterior-superior frontal cortex compared with treated MTFs and cisgender men. However, both transgender groups presented lower bilateral insular gray matter volumes than the cisgender women group. Given that the insula is linked to body awareness, one possible interpretation of these results is that this difference in insular volume could be characteristic of MTFs and related to the neural network of body perception, reflecting the distress associated with GD.

Longitudinal studies. Six studies examining the long-term effects of GAHT on the brain structure of transgender individuals (Table 2) have been published [107-112]. Overall, these morphological studies have shown that GAHT either feminizes brain structures in MTFs or defeminizes brain structures in FTMs.

In MTFs, 4 months of anti-androgen and estrogen GAHT resulted in decreased brain volumes and increased ventricle volumes "toward female proportions" compared with male controls $[108,111]$. This decrease in brain volume was ten times more than the average annual decrease in non-transgender adults [111], occurred specifically in the right hippocampal region, and was correlated with changes in progesterone levels [108]. Conversely, FTMs in the Hulshoff Pol et al. [111], study displayed an increase in total brain and hypothalamic volume and decreased ventricle volumes toward male proportions compared with female controls after 4 months of androgen GAHT. These results suggest plastic changes of the transgender adult human brain structure, specifically subcortical structures related to memory and emotional processing, toward the typical size of the identified gender under the influence of GAHT.

Similarly, diffusion tensor imaging (DTI) studies showed that GAHT alters white matter microstructure in FTMs such that they become more like cisgender males and less like their cisgender female counterparts $[107,110,112]$. After at least 7 months of $\mathrm{GAHT}$, fractional anisotropy (FA) values - a measure of connectivity - increased in the right superior longitudinal fasciculus (SLF) and the right corticospinal tract (CST) compared with pretreatment values among 15 FTMs [107]. This suggests that prescribed exogenous hormones mimic endogenous hormone 
Table 3. GAHT effects on neuronal activation (fMRI)

\begin{tabular}{|c|c|c|c|}
\hline Author & Participants (\# mos GAHT) & Imaging details & Results \\
\hline \multicolumn{4}{|c|}{ Cross-sectional study design } \\
\hline Carrillo et al. [115] & $\begin{array}{l}-23 \mathrm{MC} \\
-19 \mathrm{FC} \\
-18 \mathrm{MTF} \\
-19 \mathrm{FTM}\end{array}$ & $\begin{array}{l}3 \text { Tesla magnet } \\
\text { Mental rotation task: stimuli chosen from } \\
\text { Vandenberg-Kuse MRT Check List }\end{array}$ & $\begin{array}{l}\text { - FTMs on GAHT showed no specific pattern of } \\
\text { cerebral activation. } \\
\text { - MTFs on GAHT showed a specific pattern of cerebral } \\
\text { activation during a 3D mental rotation task that } \\
\text { differed from MCs in the parietal region and from FCs } \\
\text { in the orbital prefrontal region. } \\
\text { - A negative correlation in MTFs was found between } \\
\text { the number of months of GAHT and the cerebral } \\
\text { activation in the parietal, occipital, and temporal } \\
\text { regions. }\end{array}$ \\
\hline Schöning et al. [114] & $\begin{array}{l}\text { - } 11 \text { MTF (untreated) } \\
\text { - } 11 \text { MTF (during GAHT) } \\
-11 \text { MC }\end{array}$ & $\begin{array}{l}3 \text { Tesla magnet } \\
\text { Control: passive viewing of same 3D } \\
\text { objects non-rotated/in identical } \\
\text { projection) } \\
\text { Activation (mental rotation task: classical } \\
\text { 3D objects from standard mental rotation } \\
\text { test) }\end{array}$ & $\begin{array}{l}\text { - The activation differences in key mental rotation } \\
\text { areas in MCs compared with untreated MTFs and } \\
\text { MTFs undergoing GAHT were largely independent of } \\
\text { GAHT. In other words, the brain activation pattern for } \\
\text { the mental rotation task did not seem to be } \\
\text { significantly affected by hormonal therapy. } \\
\text { - No significant differences in mental rotation } \\
\text { performance were found between MCs, untreated } \\
\text { MTFs, and treated MTFs after } 6 \text { months of GAHT. } \\
\text { - Both treated and untreated MTFs more often used } \\
\text { verbalization (typically associated with females) as a } \\
\text { strategy for solving mental rotation problems. } \\
\text { Both untreated MTFs and treated MTFs showed } \\
\text { greater temporo-occipital activation than MCs. }\end{array}$ \\
\hline \multicolumn{4}{|c|}{ Longitudinal study design } \\
\hline Sommer et al. [118] & $\begin{array}{l}-8 \text { MTF (pre and post } \\
3 \text { months GAHT) } \\
-6 \text { FTM (pre and post } \\
3 \text { months GAHT) }\end{array}$ & $\begin{array}{l}1.5 \text { Tesla magnet } \\
2 \text { language tasks: (1) paced verb- } \\
\text { generation task with covert articulation; } \\
\text { (2) paced categorical decision task } \\
\text { Mental rotation task }\end{array}$ & $\begin{array}{l}\text { - Cerebral activation during language and mental } \\
\text { rotation tasks may be affected by GAHT. } \\
\text { - Activation during the language tasks increased after } \\
\text { three months of GAHT in both MTFs and FTMs. Total } \\
\text { language task related activity in both MTFs and FTMs } \\
\text { was correlated with post-GAHT estradiol levels. } \\
\text { - Activation during the mental rotation task did not } \\
\text { increase during GAHT, but post-GAHT testosterone } \\
\text { levels were associated with total activation during the } \\
\text { mental rotation task. } \\
\text { - Cerebral lateralization of language and spatial } \\
\text { functioning was not affected by GAHT. }\end{array}$ \\
\hline Nota et al. [119] & $\begin{array}{l}-21 \text { FTM (4 months GAHT) } \\
\text { - } 13 \text { MTF (4 months GAHT) } \\
\text { - } 20 \text { FC } \\
-17 \mathrm{MC}\end{array}$ & $\begin{array}{l}3 \text { Tesla Magnet } \\
\text { resting state functional connectivity }\end{array}$ & $\begin{array}{l}\text { - Similar functional connectivity patterns were found } \\
\text { across groups within the default mode network } \\
\text { (DMN), salience network (SN), and left working } \\
\text { memory network (WMN). } \\
\text { - Within the right WMN, MCs had significantly greater } \\
\text { functional connectivity in the right caudate nucleus } \\
\text { compared with FCs. } \\
\text { - No sex difference in functional connectivity was } \\
\text { found between MTFs and FTMs. Also, MTFs and FTMs } \\
\text { did not differ significantly from either MCs or FCs. } \\
\text { - GAHT (in FTMs and MTFs) and circulating sex steroid } \\
\text { levels (in MCs and FCs) did not affect functional } \\
\text { connectivity. }\end{array}$ \\
\hline
\end{tabular}

GAHT gender-affirming hormone therapy, GDF gender dysphoric female, GDM gender dysphoric male, MTF male-to-female transgender, FTM female-to-male transgender, $M C$ cisgender male control, $F C$ cisgender female control 
Table 4. GAHT effects on cognitive performance

\begin{tabular}{lll}
\hline Author & Participants & Measures \\
Cross-sectional study design & \\
Miles et al. [124] & -29 MTF (estrogen treated) & - Wechsler Memory Scale (Digit Span and \\
& -30 MTF (untreated) & Paired Associate Learning subtests) \\
& - Mental rotations \\
& - Controlled associations \\
& - Vocabulary
\end{tabular}

Results

- Estrogen treated MTFs scored higher on Paired Associate Learning compared with the untreated MTFs. Possible influence of estrogen in MTFs on verbal memory tasks, but cross-sectional design limits interpretation of causality.

- No differences between the treated and untreated MTFs were detected on the Digit Span (control memory) task or on other cognitive tasks, including Mental Rotations, Controlled Associations, and Vocabulary.

Gómez-Gil et al. - 9 FTM (untreated) - Verbal memory tests (Logical Memory, [125]

- 10 FTM (androgen treated)

Verbal Paired Associates immediate and delayed recall)

- Visual memory tests (Visual Paired Associates immediate and delayed recall, Rey-Osterrieth Complex Figure Test copy and - Androgen treated FTMs also achieved delayed recall)

- Functional lateralization (handedness, functional cerebral lateralization)

- Perceptual speed test (female-biased)

- Map memory test

- Mental rotations test (male-biased)

Longitudinal study design

Gómez-Gil et al. - 14 FTM (6 months GAHT) [125]

Paap and Haraldsen All tested at baseline, after [130] 3 months, after 12 months $-15 \mathrm{FC}$

$-14 \mathrm{MC}$

-21 FTM

- 12 MTF

Slabbekoorn et al. - 20 MTFs (18 months anti[127] (20) - 25 FTMs (18 months testosterone) Rey-Osterrieth Complex Figure Test copy and

Seven tests selected with established sex

- Androgen treated FTMs performed
- Verbal memory tests (Logical Memory, Verbal Paired Associates immediate and delayed recall)

- Visual memory tests (Visual Paired Associates immediate and delayed recall, delayed recall)

Two subtests measuring arithmetic ability:

- Arithmetic aptitude

- Arithmetic operations differences:

1. verbal reasoning task (neutral): indication of general intelligence

2. $2 \mathrm{D}$ rotated figures (favor $\mathrm{MC}$ ): measure spatial ability

3. 3D rotated figures (favor $\mathrm{MC}$ ): measure spatial ability

4. hidden figures (favor MC)

5. verbal fluency-words + verbal fluencysentences (favor FC)

6. fine motor movement (favor FC): dexterity fine motor behavior, complex finger movements

7. perceptual speed (favor FC) significantly better than untreated FTMs on Visual Paired Associates immediate recall and Rey-Osterrieth Complex Figure (ROCF) Test delayed recall.

significantly higher scores for copy accuracy on the ROCF.

- No significant differences were found between groups for any verbal memory test.

- MTFs demonstrated less exclusive righthandedness compared with MCs.

- No significant differences between MTFs and MCs were observed on any of the cognitive tasks.

- FTMs only improved significantly on the Visual Paired Associates immediate recall task after 6 months of androgen GAHT.

- There were no significant differences on any verbal memory test (Logical Memory, Verbal

Paired Associates immediate and delayed recall), nor on any measure of the Rey-Osterrieth Complex Figure Test (copy and delayed recall).

- At baseline, MTFs and MCs left an equal number of items unanswered. But during retest, MCs left fewer items unanswered that MTFs. - No significant difference was found between FCs and FTMs at any time point.

- For FTMs, testosterone had an enhancing and not quickly reversible effect (even 5 weeks after termination) on 3D spatial ability performance over 1.5 years. There was no deteriorating effect on verbal fluency.

- For MTFs, anti-androgen and estrogen therapy had no declining effect on 3D spatial ability. There was no enhancing effect on verbal fluency. - On the 3D spatial task, untreated MTFs initially perform better than untreated FTMs. After 3 months of GAHT, MTF and FTM performance is roughly equal. After 10 months of GAHT, MTFs perform worse than FTMs, showing that testosterone enhances visuospatial ability, while estrogen suppresses visuospatial ability. - No hormonal influence was found in either MTFs and FTMs on any of the following cognitive functions: verbal fluency, form recognition, fine motor mvmt, perceptual speed, hidden figures, verbal reasoning/general intelligence.

- In FTMs, 3 months of androgen GAHT were associated with an increase in spatial ability (improved Rotated Figures performance), a decrease in verbal fluency (decreased Word Production and Sentence Production scores), and no effect on verbal reasoning. 


\begin{tabular}{|c|c|c|c|}
\hline Miles et al. [133] & $\begin{array}{l}\text { Group } 1 \text { A-B ( } n=27 \text { MTF): } \\
\text { before, 3-12 after months } \\
\text { GAHT } \\
\text { Group 2 B-A ( } n=27 \text { MTF): } \\
\text { On GAHT, } 8 \text { weeks after stop } \\
\text { GAHT } \\
\text { Group } 3 \text { ( } n=20 \text { MTF): on } \\
\text { GAHT, 3-12 months later }\end{array}$ & $\begin{array}{l}\text { Cognitive tasks showing sex differences } \\
\text { favoring } \mathrm{F} \text { (VERBAL FLUENCY): verbal fluency, } \\
\text { controlled associations } \\
\text { Cognitive tasks showing sex differences } \\
\text { favoring } \mathrm{M}(\mathrm{VISUAL-SPATIAL)}: \text { mental } \\
\text { rotations, judgment of line angle and } \\
\text { position, memory tasks } \\
\text { Memory tasks showing sex differences } \\
\text { favoring } \mathrm{F} \text { (VERBAL MEMORY): verbal } \\
\text { memory tests (visual PAL, object memory, } \\
\text { location memory) } \\
\text { Memory tasks showing sex differences } \\
\text { favoring } \mathrm{M} \text { (VISUAL MEMORY): visual } \\
\text { reproduction, figural memory, visual memory } \\
\text { span }\end{array}$ & $\begin{array}{l}\text { Three to } 12 \text { months of estrogen GAHT for MTFs } \\
\text { has little or no influence on sex-typed aspects of } \\
\text { cognition (visuospatial, verbal performance) or } \\
\text { memory. }\end{array}$ \\
\hline
\end{tabular}

GAHT gender-affirming hormone therapy, GDF gender dysphoric female, GDM gender dysphoric male, MTF male-to-female transgender, FTM female-to-male transgender, $M C$ cisgender male control, $F C$ cisgender female control

effects on brain structure as previous work has shown that the right SLF, the forceps minor, and the CST of cisgender males are characterized by significantly higher FA values than those of cisgender females. Interestingly, greater increases in FA values in the SLF and CST were associated with higher free testosterone index values in FTMs even before beginning a course of GAHT, leading to the conclusion that the right SLF in FTMs is inherently masculinized prior to hormone treatment. Testosterone administration further increases (masculinizes) the FA values in the SLF. Likewise, testosterone GAHT in FTMs has been found to change adult hypothalamic microstructure and potentially related neural activity toward "male" patterns [112]. Testosterone-related 
reductions in mean diffusivity were observed in the lateral hypothalamus, with significant reductions occurring unilaterally after 1 month and bilaterally after 4 months of GAHT. These reductions in mean diffusivity were significantly correlated with GAHT-induced increases in free androgen index and bioavailable testosterone. Additionally, $14.4 \pm 6$ months of masculinizing GAHT in 22 FTMs was found to increase FA in the posterior portion of the fronto-occipital tract connecting the medial prefrontal cortex (mPFC) and occipital cortex, as well as increase functional connectivity between the MPFC and temporo-parietal junction [110]. Therefore, testosterone GAHT seems to result in functional changes in brain areas potentially linked to self-referential and own-body perception.

In FTMs, at least 6 months of testosterone therapy also increases cortical thickness in posterior regions of the cortex [109], increases bilateral cortical thickness in the insular and superior cortices, and changes the cortico-cortical thickness covariation between the mPFC and occipital cortex [110]. This may indicate some coordinated, testosterone-induced "structural connectivity" changes between frontal, occipital, and temporal regions in FTMs [110]. There is also a significant positive correlation between the serum testosterone and free testosterone index changes and cortical thickness changes in the parieto-occipito-temporal regions among these FTM individuals, as reported by ZubiaurreElorza et al. [109]. However, Burke et al. [110] described that the mPFC in FTMs remained unchanged after GAHT, even though the mPFC was thicker in FTMs than in both FCs and MCs to begin with. In MTFs, at least 6 months of estrogen and anti-androgen therapy is associated with decreased cortical thickness, decreased subcortical volumetric measures, and an enlargement of the ventricular system [109].

Taken together, the above six studies suggest that even relatively short-term administration of GAHT influences the gross morphology and white matter microstructure of the brain in such a manner that trans individuals become more like their identified gender with respect to these brain outcomes. Additional research would need to elucidate the specific mechanisms behind these changes as well as the clinical consequences of using GAHT for years instead of months.

GAHT effects on neuronal activation

Cross-sectional studies. Three cross-sectional studies in adults assessed brain activation patterns using $\mathrm{fMRI}$ in response to GAHT [113-115]. No pre-GAHT baseline measures were obtained and these studies focused primarily on tasks for which there is a known sex-bias in either performance or brain response. Both performance differences as well as brain activation differences between groups are highlighted in Table 3. In a four-group study which included cisgender and transgender male and female adults, cisgender males performed more accurately on a 3D mental rotation task than cisgender females, as expected [115]. Surprisingly, performance as assessed by reaction time among FTMs using high dose testosterone was slower than that of cisgender females suggesting that for this measure, testosterone treatment did not make FTMs more consistently "masculine" in their performance. Again, baseline behavioral differences in performance cannot be ruled out, but worse performance in the testosterone treated group is of concern and should be evaluated using a longitudinal study design.

In this same study, brain activation outcomes as measured by the blood oxygen level dependent (BOLD) signal during performance of the mental rotation tasks suggests that trans and cisgender individuals differ in several brain regions when trans individuals have undergone GAHT. Typically, parietal activation during task performance differs by sex, with greater activation among cisgender males than cisgender females [116, 117]. Although the expected occipito-parietal-frontal pattern of brain activation during mental rotation was observed in all groups, parietal activation was less in MTF individuals compared with cisgender males. In addition, number of months of GAHT was negatively correlated with parietal activation among MTF participants. While it is tempting to conclude from these data that GAHT "de-masculinizes" parietal activation, one must remember that these differences between cis and trans $X Y$ chromosome individuals could have been present at baseline, prior to GAHT. Interestingly, FTM individuals in this study did not differ from either control group. In a smaller study of MTFs using estrogen, MTFs not using estrogen, and cisgender male controls, there were no group differences in BOLD signal during task performance. However, MTFs relied on verbal strategies for task performance to a greater degree than cisgender males, providing additional support for baseline differences in cognition between MTFs and cisgender male controls. This finding is further indication that transgender individuals have cognitive processes that align with their gender identity rather than natal sex. In contrast, those in both transgender groups showed greater temporo-occipital activation than male controls [114]. These findings are based upon 11 individuals in each group and must therefore be considered preliminary and interpreted with caution.

In a small cross-sectional study of transmen and women using long-term GAHT (FTM for a mean duration of 84.52 months, MTF for 81.56 months) compared with cisgender men and cisgender women, differences between FTMs and cisgender female controls were reported on both intensity of spontaneous resting-state activity and local synchronization of specific brain areas in the frontal cortex, medial temporal lobe, and cerebellum [113]. For FTMs, resting-state activity in the cerebellum and frontal cortex was correlated with circulating androgen levels.

Longitudinal studies. Again, longitudinal studies of GAHT on brain activation during task performance (Table 3 ) are uncommon and focus solely on tasks with known sex differences in performance (i.e., verbal fluency and mental rotation). Sommer et al. [118] reported that 3 months of GAHT may influence cerebral activation patterns during language and mental rotation tasks in transgender individuals ( $\mathrm{MTF}=8, \mathrm{FTM}=6$ ), but lateralization of language and spatial functioning is stable. The total languagerelated activation increased after GAHT compared with baseline in both FTMs and MTFs $(p=0.08)$, and correlated to post-treatment serum estradiol levels $(p=0.05)$. However, activation during mental rotation did not increase during treatment $(p=0.34)$, but post-GAHT testosterone levels correlated with total activation during mental rotation $(p=0.01)$. The investigators did not conduct whole brain analyses of effects of sex steroid treatment on activation patterns for either task, but instead focused on taskactivated ROls.

Another study also focused on whether activation patterns in trans people were either more sex-typical or sex-atypical, but this time with respect to resting state functional connectivity patterns [119]. Similar functional connectivity patterns were found in 21 FTMs, 13 MTFs, 20 cisgender female controls, and 17 cisgender male controls within the default mode (DMN), salience (SN), and working memory (WMN) networks. However, cisgender males displayed significantly greater functional connectivity in the right caudate nucleus within the right WMN compared with cisgender females. MTFs and FTMs did not differ significantly in functional connectivity, and they did not differ significantly from either of the cisgender groups as well. Functional connectivity also did not appear to be affected by 4 months of GAHT in MTFs and FTMs or by circulating sex steroids in the cisgender individuals. Therefore, it is possible that the right WMN undergoes sexually dimorphic differentiation between cisgender males and females, but this differentiation is reduced in transgender individuals.

Performance on measures of empathy and emotional processing are also known to vary by sex [120-122]. Empathy scores and resting state functional connectivity ( $\mathrm{rsFC}$ ) measurements were 
examined in male and female controls as well as both MTFs and FTMs prior to, and after 4 weeks and 4 months of GAHT [123]. MTFs differed at baseline from all other groups in a rsFC network around the supramarginal gyrus, a region important for interpersonal emotion processing, but became more similar to the other groups over the course of GAHT administration. While taking GAHT was not correlated with rsFC in this network, a GAHTindependent association between empathy scores and rsFC was found.

\section{GAHT effects on cognitive performance}

Cross-sectional studies. Estrogen therapy in MTFs has been shown to impact performance on some (paired associate learning: immediate and delayed recall), but not all (mental rotation, controlled associates) tasks for which there are typical sex differences and in the direction that one would expect. No effect was observed on at least one task (digit symbol) for which there is no gender bias among cisgender individuals (Table 4) [124]. In another sample, no differences in mental rotation performance were observed between cisgender men, MTF individuals naïve to GAHT, and MTF individuals after at least 6 months of GAHT (Table 3) [113]. However, definitive conclusions from this study are limited given its small sample size, cross-sectional methodology, and lack of cisgender female control group.

FTM transgender individuals on androgen treatment for at least 6 months performed better than FTM subjects off treatment on the same two visual memory tasks, but no differences were noted for a verbal memory test [125]. Like the studies above, this finding signifies some correlation between GAHT and alignment of performance to gender identity as opposed to natal sex. While their performance on the male-biased task improved, no negative effect was observed for performance on the female-biased task.

With regard to cerebral lateralization, which has been shown to vary between the sexes in cis-gender populations, MTF transgender subjects who received at least 3 months of GAHT demonstrated less exclusive right-handedness as opposed to cisgender control males, but did not differ significantly in cognitive tests that have been shown to favor one sex over the other (identical pictures, 3D mental rotation, building memory). No group differences were found on the visual-split-field or cognitive tasks [126]. The cognitive test battery included three sex-specific tasks: a female-biased perceptual speed test, a map memory test, and a male-biased mental rotations test. Furthermore, there are no direct associations between endocrine measures (elevated estradiol and suppressed testosterone concentrations), lateralization, and cognition. However, this study suffers from methodological missteps, as it did not control for demographic characteristics such as age at transition, sexual orientation, or marital status. Furthermore, the data would have been stronger had untreated MTF transgender individuals been used as controls instead of cisgender males.

Longitudinal studies. Cognitive performance also seems to be affected by GAHT. Most studies (Table 4) suggest that GAHT in FTMs enhances performance on visual memory [125] and 3D spatial memory tasks $[127,128]$, and worsens verbal fluency [128, 129] and arithmetic answering strategies [130].

A small sample of FTM transgender participants $(n=14)$ tested before and after 6 months of androgen treatment showed significant improvement in their performance on a visual memory task, a skill that generally tends to favor males [125]. However, androgen GAHT did not have any influence on verbal memory, which is a domain that typically favors females. This indicates that androgens may have an activational effect on visual memory in FTM transgender individuals, causing greater alignment with the performance of their identified gender. However, this study lacked an untreated transgender control group for comparison.
An investigation on whether GAHT influenced test answering strategy found no baseline differences between untreated gender dysphoric (GD) males and cisgender male controls in number of questions left blank on an arithmetic test. In subsequent retests 3 and 12 months post-GAHT initiation for the GD group, cismale controls appeared to gain confidence and leave fewer items unanswered than the transgender group, indicating that GAHT is associated with a lack of adjustment in answering strategy in MTF individuals. No difference was found between FTM individuals and cisgender control females at any time point [131].

A pronounced effect of androgen treatment was found on spatial ability in FTM subjects over a period of 18 months [127]. Untreated MTF individuals originally performed better on visuospatial tasks than untreated FTMs, reflecting the typical male bias on spatial ability. However, after about 10 months of GAHT, the sex difference reversed, with both groups performing more in line with their identified gender. This study demonstrates that testosterone has an enhancing effect on spatial ability performance, but no deteriorating effect on verbal fluency in FTMs.

There is further evidence that androgens quickly affect cognitive performance in FTM individuals [128]. For example, enhanced spatial ability and deteriorating verbal fluency are clearly associated with as little as 3 months of androgen treatment, though there was no control group for comparison in this sample of 22 FTM participants. Later, this study followed up with an even bigger sample of FTM and MTF transgender individuals and worked with a large battery of tests on aggression, sexual motivation, and cognitive functioning [129]. Administration of androgens to FTMs over 3 months was strongly associated with an increase in aggression, sexual arousal, and spatial ability, as well as a decrease in verbal fluency. Conversely, androgen deprivation in MTFs was linked to a decrease in anger and aggression, sexual arousal, and spatial ability, as well as an increase in verbal fluency. These findings offer support for the hypothesis that GAHT can affect gender-specific behaviors, likely as a result of specific brain changes.

Studies on the cognitive performance effects of GAHT overwhelmingly support the gender-affirming effect on cognitive tasks that have shown well-established sex differences. Androgens enhance "male-like" characteristics among FTMs and are associated with decreases in performance on typically male-dominated cognitive tasks among MTFs. In contrast, longitudinal studies suggest that the opposite is true for hormone manipulations used by males transitioning to female. MTFs show a reduction in performance on 3D visuospatial tasks $[127,129]$ and increased verbal fluency. Relatively few studies have concluded that GAHT results in no change in verbal or visual spatial performance among those transitioning from one gender to the other $[25,131,132]$.

No differential effect on six cognitive abilities (perception, arithmetic, rotation, visualization, logic, and verbalization) was found between untreated cisgender control subjects and gender dysphoric patients tested before initiating GAHT and 3 and 12 months after [131]. Both the gender dysphoric individuals and controls show an identical time-dependent improvement in cognitive performance, perhaps implying that GAHT does not change sex-sensitive cognitive performance in gender dysphoric patients. However, this conclusion is weakened by the fact that the study used cisgender individuals as the control rather than untreated gender dysphoric patients.

In MTF individuals, there is reportedly no evidence that duration or dosage of treatment over the course of 3-12 months was related to any verbal fluency, visual-spatial, verbal memory, and visual memory outcomes [132]. These results suggest that MTF transgender patients do not need to anticipate any appreciable changes in sex-typed aspects of memory or cognitive function due to estrogen treatment.

Van Goozen et al. [25] reported evidence of an organizing effect of sex hormones on cognitive functioning and visuospatial ability in 
transgender individuals. Performance on four different visuospatial tests revealed a linear increase in accuracy from cisgender female controls, to FTMs (before GAHT), to MTFs (before GAHT), and finally to cisgender male controls. Furthermore, after 14 weeks of GAHT, there was no effect of sex hormones on the visuospatial ability of transgender individuals, highlighting the lack of activational effects of short-term GAHT with respect to this particular cognitive task. Using a medium-sized sample of $\sim 20$ for each transgender group, this study used standardized levels of GAHT, which makes for more reliable conclusions across individuals.

GAHT effects on psychology and behavior

Studies of mental health among transgender people consistently report alarmingly elevated rates of anxiety, depression, and suicide risk [11, 133-138]. Though behavioral health risks generally decline with GAHT [102], risk remains elevated above that of cisgender individuals matched for age, natal sex, and new assigned sex [138]. No consensus has been reached regarding the sex specificity of psychopathology; while some studies report higher incidence of psychopathology in transgender women relative to transgender men $[125,140,141]$, one study suggested the opposite [142]. These symptoms of psychopathology are thought to be the result of distress stemming from incongruence between appearance and gender identity, as well as social rejection - prejudice, discrimination, and stigma [134, 143]. Consequently, many transgender people face greater socioeconomic barriers and disproportionately higher rates of sexual abuse and assault.

Although being trans is associated with increasing mental health problems due to social circumstances, there is now growing evidence suggesting that gender-affirming social support and GAHT are linked to better mental health outcomes. A 2-year follow-up study observed a significant reduction $(p<0.01)$ in body dissatisfaction and GD after 3 months of GAHT in MTFs and FTMs, as measured by the Body Uneasiness Test and Gender Identity/GD Questionnaire. In addition, 12 months of GAHT is significantly associated with psychopathology reduction $(p<0.001)$ as assessed by the Symptoms Checklist (SCL-90R) [144]; significantly lower self-reported anxiety, depression, and global psychological symptoms, as well as psychiatric distress and functional impairment [11]; and greater self-esteem, less severe depression symptoms, and higher "psychological-like" dimensions of quality of life (psychological wellbeing and taking care of oneself) [145]. The degree to which these effects of GAHT are secondary to receiving social and medical support for one's decision to transition, enhancing congruence between gender identity and physical appearance, or modulating brain neurochemistry, structure and function with exogenous sex steroids is not known.

Though GAHT is thought to enhance overall well-being, there are different effects of estrogen versus testosterone therapies. Androgen GAHT in FTMs has been shown to significantly enhance anger proneness, aggressive tendencies, sexual arousability, and motivation, while feminizing treatment is associated with the reverse in MTFs [129]. Anti-androgenic therapy, using the pure antiandrogen nilutamide (Anandron ${ }^{\circledR}$, Sanofi Aventis) and no estradiol, reduced sexual arousal and sexual desire after 8 weeks in MTFs [146]. Nilutamide functions by blocking the biological effects of androgens produced by the organism through competition at the level of hormone receptors in androgen-sensitive tissues (skin, prostate, pituitary, central nervous system, etc.). MTFs receiving 12 or more months of estradiol and progesterone were less likely to exhibit scores reflective of psychopathology on the Minnesota Multiphasic Personality Inventory (MMPI) compared with untreated gender dysphoric males [147]. Longer periods of feminizing treatment are associated with greater emotional adjustment scores on the MMPI.

In summary, the safety of GAHT and efficacy with respect to improving psychological well-being is encouraging, particularly for
MTFs. However, this literature is limited greatly by studies with small sample sizes and extensive variations in methodology (i.e., age at which GAHT was initiative, duration of treatment, pharmacologic regimen, documentation of hormone levels post GAHT).

\section{Summary and future research directions}

The extant literature describing neuroimaging outcomes among transgender populations focuses primarily on questions of how similar the brain of the transgender individual is to their natal sex and whether use of GAHT enhances similarity between the brain of their natal sex and that of their desired gender. Most, but not all studies, indicate that the transgender individual is more similar to their preferred gender with respect to cerebral and gray matter volume and performance on gender-biased cognitive tasks (e.g., verbal and spatial tasks). There is a signal for GAHT to enhance the transgender individuals' similarity to their preferred gender.

While these outcomes may have been of interest in previous decades, this information is not particularly useful or relevant to most transgender individuals and their health care providers with respect to their day-to-day quality of life. As discussed earlier, the prefrontal cortex - the site of estrogen modulation of executive function - is necessary for cognitive processes such as sustained attention, working memory, organization, and planning. It is well known that premature loss of estradiol among cisgender women leads to significant risk for medical comorbidities of aging including increased risk for executive dysfunction [18] and dementia [63]. Whether the same is true for FTM individuals is not known, but high dose testosterone variably diminishes endogenous estradiol production in FTMs, leaving the individual in a relative hypoestrogenic state. Therefore, the long-term effects of hypoestrogenism in the context of hypertestoteronism should be investigated. If the logic behind PCOS and lower levels of estrogen post-menopause were applied to current GAHT, then one would expect that suppression of estrogen levels and increased testosterone levels in FTM individuals would impact cognition and possibly adversely impact psychological well-being, increasing irritability and impulsivity [73]. While cisgender males aromatize testosterone to estradiol in the CNS [148], it is not known whether FTM transgender individuals also aromatize testosterone to estradiol sufficiently to estrogenize the brain. Finally, it is possible that the organizational effects of gonadal steroids in utero for the transgender individual would create a scenario that alters their need for various gonadal steroids for optimal brain health across the lifespan.

Currently, investigations on the neuroanatomical changes in transgender individuals after prolonged GAHT are too few in number to examine the putative effects of GAHT on neuroanatomy. To date, there are no studies assessing the effects of GAHT on functional network connectivity. Recent findings from our laboratory indicate enduring impact of childhood adversity on within network connectivity among early postmenopausal cis-gender women [17]. That estradiol treatment enhanced within network connectivity among those women with high levels of childhood adversity, but had no appreciable impact on those with low levels of childhood adversity, suggests a differential impact of gonadal steroids on brain structure and function based upon adverse exposures during critical windows of development. This would seem particularly relevant to the transgender community as their exposures to childhood adversity can be substantial.

Application of $\mathrm{fMRI}$ to the investigation of the transgender brain and effects of GAHT is in its infancy, but holds promise as a tool to determine regional and network effects of GAHT. Gonadal steroids have a profound impact on brain structure, function, regional connectivity, and neurochemistry [16] which underpin executive 
functions, affect, and reward processing. It is critical that future research considers these cognitive domains and focus substantially less on "sexually dimorphic" cognitive processes which typically show sex differences among cisgender individuals. We would argue that learning and memory, the capacity to organize, focus and manage one's affect as well as appreciate the rewarding nature of appetitive stimuli is critical to long-term health and quality of life. Special attention should be paid to effects of GAHT on the neural correlates of behavior during the transition process since research to date has not examined emotional or affective responding despite high rates of affective disorders in this population.

The currently available structural MRI studies also contain small numbers of transgender adults and even fewer transgender adolescents. Given the profound impact of post-pubertal levels of and fluctuations in gonadal steroids on brain structure and function, data obtained from transgender adults who are many years post-puberty should not be extrapolated to adolescents who choose to block their transition to puberty. Similarly, individual factors such as socioeconomic status, race/ethnicity, education, exposures to childhood adversity, and lifetime stress or trauma could dramatically impact central nervous system function during critical windows of development leading to risk or resilience to cognitive and mood changes during hormonal manipulations [2, 3, 149].

Furthermore, most studies to date have been cross-sectional in nature, included small samples, and examined the effects of short-term GAHT ( $\leq 18$ months), though most individuals will use GAHT for decades. There are no randomized controlled trials of GAHT. Therefore experimental, longitudinal studies (>18 months) must be prioritized, using neuroimaging methods to focus on the effects of GAHT on brain regions/networks directly related to accomplishing daily tasks, to allow direct comparison between baseline and post-GAHT values. And given that the current literature has almost exclusively treated gender as a binary thus far (e.g., a person can be either male, female, FTM, or MTF), future studies should consider gender as a spectrum to be more inclusive to those who are transgender, gender non-binary, gender non-conforming, gender fluid, etc. In particular, research should seek to include transfeminine and especially transmasculine individuals. Considering the majority of transgender research studies thus far have looked at MTFs, there are many unanswered questions regarding the potential impact of high dose testosterone on long term brain health of FTM and transmasculine patients, with significant causes for concern, as mentioned above.

With more people identifying as transgender and requesting GAHT, the field of transgender health needs to be able to provide better information about the potential brain effects of long-term GAHT. Importantly, the bulk of the data suggests that self-reported level of function and quality of life is improved with GAHT, though still below that of cisgender individuals. The precise clinical relevance of neuroanatomical changes after GAHT remains unknown. Characterizing the different modulations associated with GAHT is critical, considering reports of physiological risk with hormonal treatment and rates of psychopathology in transgender individuals. Future studies are required to replicate previous findings and examine whether these neuroanatomical differences are associated with functional or cognitive changes. In summary, we recommend that upcoming brain imaging studies examine long-term (years) use of GAHT, study sufficiently large samples to address potential modifiers of hormone response (e.g., early life stress, hormone levels), focus on cognitive domains crucial for executive function, broaden gender definitions beyond binarized male and female identities, and enroll more FTM and transmasculine individuals.

\section{ACKNOWLEDGEMENTS}

The authors wish to recognize the following funding sources related to the writing of this manuscript: National Institute of Mental Health (NIMH) and Office of Research on Women's Health (ORWH; P50 MH-099910; CNE); Office of Research on Women's Health (K12 HD085848); NIMH (K23 MH107831, LH), NIMH (K23 MH102360, SK) and the Emily Hartshorne Mudd Research Fellowship (HBN) and the University of Pennsylvania Center for Undergraduate Research (HBN).

\section{ADDITIONAL INFORMATION}

Competing interests: The authors declare no competing interests.

Publisher's note: Springer Nature remains neutral with regard to jurisdictional claims in published maps and institutional affiliations.

\section{REFERENCES}

1. Human Rights Campaign. (n.d.). Glossary of terms. http://www.hrc.org/resources/glossary-of-terms. Accessed 16 June 2017.

2. Bale TL, Epperson CN. Sex as a biological variable: who, what, when, why, and how. Neuropsychopharmacology. 2017;42(2):386-96.

3. Bale TL, Epperson CN. Sex differences and stress across the lifespan. Nat Neurosci. 2015;18(10):1413-20.

4. Mueller SC, De Cuypere G, T'Sjoen G. Transgender research in the 21st century: a selective critical review from a neurocognitive perspective. Am J Psychiatry. 2017;174(12):1155-62

5. Flores AR, Herman JL, Gates GJ, Brown TN. How many adults identify as transgender in the United States? The Williams Institute. 2016.

6. Hansbury $G$. The middle men: an introduction to the transmasculine identities. Stud Gend Sex. 2005;6(3):241-64.

7. American Psychiatric Association. Diagnostic and statistical manual of mental disorders. 3rd ed. Arlington, VA: American Psychiatric Publishing; 1980.

8. American Psychiatric Association. Diagnostic and statistical manual of mental disorders. 4th ed. Arlington, VA: American Psychiatric Publishing; 1994.

9. American Psychiatric Association. Diagnostic and statistical manual of mental disorders. 5th ed. Arlington, VA: American Psychiatric Publishing; 2013.

10. Glicksman E. Transgender today. Monit Psychol. 2013;44(4):36.

11. Colizzi M, Costa R, Todarello O. Transsexual patients' psychiatric comorbidity and positive effect of cross-sex hormonal treatment on mental health: results from a longitudinal study. Psychoneuroendocrinology. 2014;39:65-73.

12. Heylens G, Elaut E, Kreukels BP, Paap MC, Cerwenka S, Richter-Appelt H, et al. Psychiatric characteristics in transsexual individuals: multicentre study in four European countries. Br J Psychiatry. 2014;204(2):151-6.

13. Manieri C, Castellano E, Crespi C, Di Bisceglie C, Dell'Aquila C, Gualerzi A, Molo M. Medical treatment of subjects with gender identity disorder: the experience in an Italian public health center. Int J Transgenderism. 2014;15(2):53-65.

14. Sisk CL, Foster DL. The neural basis of puberty and adolescence. Nat Neurosci. 2004;7:1040-7.

15. Arnold AP, Breedlove SM. Organizational and activational effects of sex steroids on brain and behavior: a reanalysis. Horm Behav. 1985;19(4):469-98.

16. Shanmugan S, Epperson CN. Estrogen and the prefrontal cortex: towards a new understanding of estrogen's effects on executive functions in the menopause transition. Hum Brain Mapp. 2014;35(3):847-65.

17. Shanmugan S, Loughead J, Cao W, Sammel MD, Satterthwaite TD, Ruparel K, et al. Impact of tryptophan depletion on executive system function during menopause is moderated by childhood adversity. Neuropsychopharmacology. 2017a;42(12):2398.

18. Epperson CN, Shanmugan S, Kim DR, Mathews S, Czarkowski KA, Bradley J, et al. New onset executive function difficulties at menopause: a possible role for lisdexamfetamine. Psychopharmacol (Berl). 2015;232(16):3091-3100.

19. Seidman SN. Testosterone deficiency and mood in aging men: pathogenic and therapeutic interactions. World J Biol Psychiatry. 2003;4(1):14-20.

20. McIntyre RS, Mancini D, Eisfeld BS, Soczynska JK, Grupp L, Konarski JZ, Kennedy $\mathrm{SH}$. Calculated bioavailable testosterone levels and depression in middle-aged men. Psychoneuroendocrinology. 2006;31(9):1029-35.

21. McCarthy MM, Arnold AP, Ball GF, Blaustein JD, De Vries GJ. Sex differences in the brain: the not so inconvenient truth. J Neurosci. 2012;32(7):2241-7.

22. Bakker J, Baum MJ. Role for estradiol in female-typical brain and behavioral sexual differentiation. Front Neuroendocrinol. 2008;29(1):1-16.

23. Joel D, Berman Z, Tavor I, Wexler N, Gaber O, Stein Y, et al. Sex beyond the genitalia: the human brain mosaic. Proc Natl Acad Sci. 2015;112(50):15468-73.

24. Bao AM, Swaab DF. Sexual differentiation of the human brain: relation to gender identity, sexual orientation and neuropsychiatric disorders. Front Neuroendocrinol. 2011;32(2):214-26. 
25. Van Goozen SH, Slabbekoorn D, Gooren LJ, Sanders G, Cohen-Kettenis PT. Organizing and activating effects of sex hormones in homosexual transsexuals. Behav Neurosci. 2002;116(6):982.

26. Gómez-Gil E, Esteva I, Almaraz MC, Pasaro E, Segovia S, Guillamon A. Familiality of gender identity disorder in non-twin siblings. Arch Sex Behav. 2010;39 (2):546-52.

27. Satterthwaite TD, Vandekar S, Wolf DH, Ruparel K, Roalf DR, Jackson C, et al. Sex differences in the effect of puberty on hippocampal morphology. J Am Acad Child Adolesc Psychiatry. 2014a;53(3):341-50.

28. Yakovlev PA, Lecours IR. The myelogenetic cycles of regional maturation of the brain. In: Minkowski A, (Ed.). Regional development of the brain in early life. Oxford: Blackwell; 1967. p. 3-70.

29. Huttenlocher PR. Synaptic density in human frontal cortex - developmental changes and effects of aging. Brain Res. 1979;163:195-205.

30. Huttenlocher PR, De Courten C, Garey LJ, Van Der Loos H. Synaptic development in human cerebral cortex. Int J Neurol. 1983;16-17:144-54.

31. Satterthwaite TD, Wolf DH, Roalf DR, Ruparel K, Erus G, Vandekar S, et al. Linked sex differences in cognition and functional connectivity in youth. Cereb Cortex. 2014;25(9):2383-94.

32. Satterthwaite TD, Baker JT. How can studies of resting-state functional connectivity help us understand psychosis as a disorder of brain development? Curr Opin Neurobiol. 2015;30:85-91.

33. Shanmugan S, Satterthwaite TD. Neural markers of the development of executive function: relevance for education. Curr Opin Behav Sci. 2016; 10:7-13.

34. Gennatas ED, Avants BB, Wolf DH, Satterthwaite TD, Ruparel K, Ciric R, et al. Agerelated effects and sex differences in gray matter density, volume, mass, and cortical thickness from childhood to young adulthood. J Neurosci. 2017;37 (20):5065-73.

35. Giedd JN, Vaituzis AC, Hamburger SD, et al. Quantitative MRI of the temporal lobe, amygdala, and hippocampus in normal human development: ages 4-18 years. J Comp Neurol. 1996;366:223-30.

36. Giedd JN, Castellanos FX, Rajapakse JC, Vaituzis AC, Rapoport JL. Sexual dimorphism of the developing human brain. Prog Neuropsychopharmacol Biol Psychiatry. 1997;21:1185-201.

37. Rubia K, Russell T, Overmeyer S, Brammer MJ, Bullmore ET, Sharma T, et al. Mapping motor inhibition: conjunctive brain activations across different versions of go/no-go and stop tasks. Neuroimage. 2001;13(2):250-61.

38. Sowell ER, Thompson PM, Tessner KD, Toga AW. Mapping continued brain growth and gray matter density reduction in dorsal frontal cortex: inverse relationships during postadolescent brain maturation. J Neurosci. 2001;21 (22):8819-29.

39. Allen JS, Damasio $H$, Grabowski TJ. Normal neuroanatomical variation in the human brain: an MRI-volumetric study. Am J Phys Anthropol. 2002;118 (4):341-58.

40. Lüders $E$, Steinmetz $H$, Jäncke $L$. Brain size and grey matter volume in the healthy human brain. Neuroreport. 2002;13(17):2371-4.

41. Ruigrok AN, Salimi-Khorshidi G, Lai MC, Baron-Cohen S, Lombardo MV, Tait RJ, Suckling J. A meta-analysis of sex differences in human brain structure. Neurosci Biobehav Rev. 2014;39:34-50.

42. Cosgrove KP, Mazure CM, Staley JK. Evolving knowledge of sex differences in brain structure, function, and chemistry. Biol Psychiatry. 2007;62(8):847-55

43. Walker DL, Toufexis DJ, Davis M. Role of the bed nucleus of the stria terminalis versus the amygdala in fear, stress, and anxiety. Eur J Pharmacol. 2003;463 (1):199-216.

44. Zhou JN, Hofman MA, Gooren LJ, Swaab DF. A sex difference in the human brain and its relation to transsexuality. Nature. 1995;378(6552):68.

45. Kruijver FP, Zhou JN, Pool CW, Hofman MA, Gooren U, Swaab DF. Male-tofemale transsexuals have female neuron numbers in a limbic nucleus. J Clin Endocrinol Metab. 2000;85(5):2034-41.

46. Farhat MY, Lavigne MC, Ramwell PW. The vascular protective effects of estrogen FASEB J. 1996;10(5):615-24.

47. Scheinost D, Finn ES, Tokoglu F, Shen X, Papademetris X, Hampson M, Constable RT. Sex differences in normal age trajectories of functional brain networks. Hum Brain Mapp. 2015;36(4):1524-35.

48. Wu K, Taki Y, Sato K, Hashizume H, Sassa Y, Takeuchi $H$, et al. Topological organization of functional brain networks in healthy children: differences in relation to age, sex, and intelligence. PLOS ONE. 2013;8(2):e55347.

49. Zuo XN, Kelly C, Di Martino A, Mennes M, Margulies DS, Bangaru S, et al. Growing together and growing apart: regional and sex differences in the lifespan developmental trajectories of functional homotopy. J Neurosci. 2010;30 (45):15034-43

50. Casanova R, Whitlow CT, Wagner B, Espeland MA, Maldjian JA. Combining graph and machine learning methods to analyze differences in functional connectivity across sex. Open Neuroimag J. 2012;6:1.
51. Hjelmervik H, Hausmann M, Osnes B, Westerhausen R, Specht K. Resting states are resting traits - an FMRI study of sex differences and menstrual cycle effects in resting state cognitive control networks. PLOS ONE. 2014;9(7):e103492.

52. Miller DI, Halpern DF. The new science of cognitive sex differences. Trends Cogn Sci. 2014;18(1):37-45.

53. Gouchie C, Kimura D. The relationship between testosterone levels and cognitive ability patterns. Psychoneuroendocrinology. 1991;16(4):323-34.

54. Kimura D. Sex, sexual orientation and sex hormones influence human cognitive function. Curr Opin Neurobiol. 1996;6(2):259-63.

55. McGlone J. Sex differences in human brain asymmetry: a critical survey. Behav Brain Sci. 1980;3(02):215-27.

56. Mitchell ES, Woods NF. Cognitive symptoms during the menopausal transition and early postmenopause. Climacteric. 2011;14:252-61.

57. Epperson CN, Sammel MD, Freeman EW. Menopause effects on verbal memory: findings from a longitudinal community cohort. J Clin Endocrinol Metab. 2013;98(9):3829-38.

58. Freeman EW, Sammel MD, Boorman DW, Zhang R. Longitudinal pattern of depressive symptoms around natural menopause. JAMA Psychiatry. 2014;71 (1):36-43.

59. Shanmugan S, Loughead J, Nanga RPR, Elliott M, Hariharan H, Appleby D, et al. Lisdexamfetamine effects on executive activation and neurochemistry in menopausal women with executive function difficulties. Neuropsychopharmacology. 2017b;42(2):437.

60. Shanmugan S, Satterthwaite TD, Sammel MD, Cao W, Ruparel K, Gur RC, et al. Impact of early life adversity and tryptophan depletion on functional connectivity in menopausal women: a double-blind, placebo-controlled crossover study. Psychoneuroendocrinology. 2017c;84:197-205.

61. Epperson CN, Amin Z, Ruparel K, Gur R, Loughead J. Interactive effects of estrogen and serotonin on brain activation during working memory and affective processing in menopausal women. Psychoneuroendocrinology. 2012:37(3):372-82.

62. Cohen LS, Soares CN, Vitonis AF, Otto MW, Harlow BL. Risk for new onset of depression during the menopausal transition: the Harvard study of moods and cycles. Arch Gen Psychiatry. 2006;63(4):385-90.

63. Shuster LT, Rhodes DJ, Gostout BS, Grossardt BR, Rocca WA. Premature menopause or early menopause: long-term health consequences. Maturitas. 2010;65 (2):161-6.

64. Weiner $\mathrm{CL}$, Primeau $\mathrm{M}$, Ehrmann DA. Androgens and mood dysfunction in women: comparison of women with polycystic ovarian syndrome to healthy controls. Psychosom Med. 2004;66(3):356-62.

65. Cooney LG, Dokras A. Depression and anxiety in polycystic ovary syndrome: etiology and treatment. Curr Psychiatry Rep. 2017;19(11):83.

66. Goetsch AL, Kimelman D, Woodruff TK. Polycystic Ovary Syndrome. In: Goetsch $\mathrm{AL}$ (Ed.) Fertility Preservation and Restoration for Patients with Complex Medical Conditions. Cham: Springer; 2017. p. 231-48.

67. Teede H, Deeks A, Moran L. Polycystic ovary syndrome: a complex condition with psychological, reproductive and metabolic manifestations that impacts on health across the lifespan. BMC Med. 2010;8(1):41.

68. Ehrmann DA. Polycystic ovary syndrome. New Engl J Med. 2005;352 (12):1223-36.

69. Abbott DH, Barnett DK, Bruns CM, Dumesic DA. Androgen excess fetal programming of female reproduction: a developmental aetiology for polycystic ovary syndrome? Hum Reprod Update. 2005;11(4):357-74.

70. Deeks AA, Gibson-Helm ME, Teede HJ. Anxiety and depression in polycystic ovary syndrome: a comprehensive investigation. Fertil Steril. 2010;93(7):2421-3.

71. Himelein MJ, Thatcher SS. Polycystic ovary syndrome and mental health: a review. Obstet Gynecol Surv. 2006;61(11):723-32.

72. Hollinrake E, Abreu A, Maifeld M, Van Voorhis BJ, Dokras A. Increased risk of depressive disorders in women with polycystic ovary syndrome. Fertil Steril. 2007;87(6):1369-76.

73. Dokras A, Stener-Victorin E, Yildiz BO, Li R, Ottey S, Shah D, Epperson N, Teede H. Androgen Excess- Polycystic Ovary Syndrome Society: position statement on depression, anxiety, quality of life, and eating disorders in polycystic ovary syndrome. Fertil Steril. 2018;109(5):888-99.

74. Rassi A, Veras AB, dos Reis M, Pastore DL, Bruno LM, Bruno RV, de Ávila MA, Nardi AE. Prevalence of psychiatric disorders in patients with polycystic ovary syndrome. Comprehensive psychiatry. 2010;51(6):599-602.

75. Haring R, Völzke H, Steveling A, Krebs A, Felix SB, Schöfl C, et al. Low serum testosterone levels are associated with increased risk of mortality in a population-based cohort of men aged 20-79. Eur Heart J. 2010;31 (12):1494-501.

76. Wang C, Jackson G, Jones TH, Matsumoto AM, Nehra A, Perelman MA, et al. Low testosterone associated with obesity and the metabolic syndrome contributes to sexual dysfunction and cardiovascular disease risk in men with type 2 diabetes. Diabetes Care. 2011;34(7):1669-75. 
77. Hyde Z, Flicker L, Almeida OP, Hankey GJ, McCaul KA, Chubb SP, Yeap BB. Low free testosterone predicts frailty in older men: the health in men study. J Clin Endocrinol Metab. 2010;95(7):3165-72.

78. Joshi D, Van Schoor NM, De Ronde W, Schaap LA, Comijs HC, Beekman AT, Lips $P$. Low free testosterone levels are associated with prevalence and incidence of depressive symptoms in older men. Clin Endocrinol (Oxf). 2010;72(2):232-40.

79. Shores MM, Smith NL, Forsberg CW, Anawalt BD, Matsumoto AM. Testosterone treatment and mortality in men with low testosterone levels. J Clin Endocrinol Metab. 2012;97(6):2050-8.

80. Zubiaurre-Elorza L, Junque C, Gómez-Gil E, Segovia S, Carrillo B, Rametti G, Guillamon A. Cortical thickness in untreated transsexuals. Cereb Cortex. 2013;23 (12):2855-62.

81. Luders E, Sanchez FJ, Tosun D, Shattuck DW, Gaser C, Vilain E, Toga AW. Increased cortical thickness in male- to-female transsexualism. J Behav Brain Sci. 2012;2:357-62.

82. Simon L, Koza'k LR, Simon V, Czobor P, Unoka Z, et al. Regional grey matter structure differences between transsexuals and healthy controls - a Voxel Based Morphometry Study. PLOS ONE. 2013;8(12):e83947

83. Rametti G, Carrillo B, Gómez-Gil E, Junque C, Segovia S, Gomez A, et al. White matter microstructure in female to male transsexuals before cross-sex hormonal treatment. A diffusion tensor imaging study. J Psychiatr Res. 2011;8:1-6.

84. Kranz GS, Hahn A, Kaufmann U, Küblböck M, Hummer A, Ganger S, Lanzenberger R. White matter microstructure in transsexuals and controls investigated by diffusion tensor imaging. J Neurosci. 2014;34(46):15466-75.

85. Hahn A, Kranz GS, Kublbock M, Kaufmann U, Ganger S, Hummer A, Seiger R, Spies M, Winkler D, Kasper S, Windischberger C, Swaab DF, Lanzenberger R. Structural connectivity networks of transgender people. Cereb Cortex. 2015;25:3527-34.

86. Yokota Y, Kawamura Y, Kameya Y. Callosal shapes at the midsagittal plane: MRI differences of normal males, normal females, and GID. In: Proceedings of the 2005 IEEE engineering in medicine and biology 27th annual conference, Shangai, China; 2005. p. 3055-8.

87. Lin CS, Ku HL, Chao HT, Tu PC, Li CT, Cheng CM, et al. Neural network of body representation differs between transsexuals and cissexuals. PLOS ONE. 2014;9 (1):e85914.

88. Soleman RS, Schagen SE, Veltman DJ, Kreukels BP, Cohen-Kettenis PT, Lambalk $C B$, et al. Sex differences in verbal fluency during adolescence: a functional magnetic resonance imaging study in gender dysphoric and control boys and girls. J Sex Med. 2013;10(8):1969-77.

89. Zucker KJ, Bradley SJ. Gender identity disorder and psychosexual problems in children and adolescents. New York, NY: Guilford Press; 1995.

90. Cohen-Kettenis PT, van Goozen SH, Doorn CD, Gooren LJ. Cognitive ability and cerebral lateralisation in transsexuals. Psychoneuroendocrinology. 1998;23:631-41.

91. Hunt DD, Carr JE, Hampson JL. Cognitive correlates of biologic sex and gender identity in transsexualism. Arch Sex Behav. 1981;10:65-77. 445

92. Berglund $H$, Lindström $P$, Dhejne-Helmy C, Savic I. Male-to-female transsexuals show sex-atypical hypothalamus activation when smelling odorous steroids. Cereb Cortex. 2008;18(8):1900-8.

93. Gizewski ER, Krause E, Schlamann M, Happich F, Ladd ME, Forsting M, Senf W. Specific cerebral activation due to visual erotic stimuli in male-to-female transsexuals compared with male and female controls: an fMRI study. J Sex Med. 2009:6:440-8.

94. Junger J, Habel U, Bröhr S, Neulen J, Neuschaefer-Rube C, Birkholz P, et al. More than just two sexes: the neural correlates of voice gender perception in gender dysphoria. PLOS ONE. 2014;9(11):e111672.

95. Hoekzema $E$, Schagen SE, Kreukels BP, Veltman DJ, Cohen-Kettenis PT, Delemarre-van de Waal H, Bakker J. Regional volumes and spatial volumetric distribution of gray matter in the gender dysphoric brain. Psychoneuroendocrinology. 2015;55:59-71.

96. Luders E, Sánchez FJ, Gaser C, Toga AW, Narr KL, Hamilton LS, Vilain E. Regional gray matter variation in male-to-female transsexualism. Neuroimage. 2009;46 (4):904-7.

97. Manzouri A, Kosidou K, Savic I. Anatomical and functional findings in female-tomale transsexuals: testing a new hypothesis. Cereb Cortex. 2017;27(2):998-1010.

98. Haraldsen IR, Opjordsmoen S, Egeland T, Finset A. Sex-sensitive cognitive performance in untreated patients with early onset gender identity disorder. Psychoneuroendocrinology. 2003;28(7):906-15.

99. Forcier MM, Haddad E. Health care for gender variant or gender nonconforming children. R I Med J. 2013;96:17-21.

100. Radix AE. Medical transition for transgender individuals. In: Eckstrand $\mathrm{KL}$, Ehrenfeld JM, editors. Lesbian, gay, bisexual, and transgender healthcare. Switzerland: Springer International Publishing; 2016. p. 351-61.

101. Grant JM, Mottet LA, Tanis J, Herman JL, Harrison J, Keisling M. National transgender discrimination survey report on health and health care.
Washington, DC: National Center for Transgender Equality and the National Gay and Lesbian Task Force; 2010.

102. Olson KR, Durwood L, DeMeules M, McLaughlin KA. Mental health of transgender children who are supported in their identities. Pediatrics. 2016;137: e20153223. peds-2015

103. Gooren LJ, Giltay EJ, Bunck MC. Long-term treatment of transsexuals with crosssex hormones: extensive personal experience. J Clin Endocrinol Metab. 2008;93 (1):19-25.

104. Van Caenegem E, Wierckx K, Taes Y, Dedecker D, Van de Peer F, Toye K, et al. Bone mass, bone geometry, and body composition in female-to-male transsexual persons after long-term cross-sex hormonal therapy. J Clin Endocrinol Metab. 2012;97(7):2503-11.

105. Mueller SC, Landre L, Wierckx K, et al. A structural MRI study in transgender persons on cross-sex hormone therapy. Neuroendocrinology. 2017b;105:123-30.

106. Spizzirri G, Duran FLS, Chaim-Avancini TM, Serpa MH, Cavallet M, Pereira CMA, et al. Grey and white matter volumes either in treatment-naïve or hormonetreated transgender women: a voxel-based morphometry study. Sci Rep. 2018;8 (1):736.

107. Rametti G, Carrillo B, Gómez-Gil E, Junque C, Zubiaurre-Elorza L, Segovia S, et al. Effects of androgenization on the white matter microstructure of female-tomale transsexuals. A diffusion tensor imaging study. Psychoneuroendocrinology. 2012;37(8):1261-9.

108. Seiger R, Hahn A, Hummer A, Kranz GS, Ganger S, Woletz M, et al. Subcortical gray matter changes in transgender subjects after long-term cross-sex hormone administration. Psychoneuroendocrinology. 2016;74:371-9.

109. Zubiaurre-Elorza L, Junque C, Gómez-Gil E, Guillamon A. Effects of cross-sex hormone treatment on cortical thickness in transsexual individuals. J Sex Med. 2014;11(5):1248-61.

110. Burke SM,Manzouri AH,Dhejne C,Bergström K,Arver S,Feusner JD,Savic-Berglund I, Testosterone effects on the brain in transgender men. Cerebral Cortex. 2017;28:1582-96

111. Hulshoff Pol HE, Cohen-Kettenis PT, Van Haren NEM, Peper JS, Brans RGH, Cahn W, Schnack HG, Gooren LJG, Kahn RS. Changing your sex changes your brain: influences of testosterone and estrogen on adult human brain structure. Eur J Endocrinol. 2006;155:s107-s114.

112. Kranz GS, Hahn A, Kaufmann U, Tik M, Ganger S, Seiger R, et al. Effects of testosterone treatment on hypothalamic neuroplasticity in female-to-male transgender individuals. Brain Struct Funct. 2018;223(1):321-8.

113. Mueller SC, Wierckx K, Jackson K, et al. Circulating androgens correlate with resting-state $\mathrm{MRI}$ in transgender men. Psychoneuroendocrinology. 2016;73:91-98.

114. Schöning S, Engelien A, Bauer C, Kugel H, Kersting A, Roestel $C$, et al. Neuroimaging differences in spatial cognition between men and male-tofemale transsexuals before and during hormone therapy. J Sex Med. 2010;7 (5):1858-67.

115. Carrillo B, Gómez-Gil E, Rametti G, Junque C, Gomez Á, Karadi K, et al. Cortical activation during mental rotation in male-to-female and female-to-male transsexuals under hormonal treatment. Psychoneuroendocrinology. 2010;35 (8):1213-22.

116. Roberts JE, Bell MA. Two- and three-dimensional mental rotation tasks lead to different parietal laterality for men and women. Int J Psychophysiol. 2003;50:235-246.

117. Zacks JM. Neuroimaging studies of mental rotation: a meta-analysis and review. J Cogn Neurosci. 2008;20:1-19.

118. Sommer IEC, Cohen-Kettenis PT, van Raalten T, vd Veer AJ, Ramsey LE, Gooren LJG, et al. Effects of cross-sex hormones on cerebral activation during language and mental rotation: an fMRI study in transsexuals. Eur Neuropsychopharmcol. 2008;18:215e21.

119. Nota NM, Burke SM, den Heijer M, Soleman RS, Lambalk CB, Cohen-Kettenis PT, et al. Brain sexual differentiation and effects of cross-sex hormone therapy in transpeople: a resting-state functional magnetic resonance study. Neurophysiol Clin. 2017;47(5-6):361-70.

120. Schulte-Rüther M, Markowitsch HJ, Shah NJ, Fink GR, Piefke M. Gender differences in brain networks supporting empathy. Neuroimage. 2008;42(1):393-403.

121. Hall JA. Gender effects in decoding nonverbal cues. Psychol Bull. 1978;85(4):845.

122. McClure EB, A meta-analytic review of sex differences in facial expression processing and their development in infants, children, and adolescents. Psychol Bull. 2000;126:424-53.

123. Spies M, Hahn A, Kranz GS, Sladky R, Kaufmann U, Hummer A, et al. Gender transition affects neural correlates of empathy: A resting state functional connectivity study with ultra high-field $7 T$ MR imaging. Neuroimage. 2016;138:257-65.

124. Miles C, Green R, Sanders G, Hines M. Estrogen and memory in a transsexual population. Horm Behav. 1998;34(2):199-208. 
125. Gómez-Gil E, Trilla A, Salamero M, Godás T, Valdés M. Sociodemographic, clinical, and psychiatric characteristics of transsexuals from Spain. Arch Sex Behav. 2009;38(3):378-92.

126. Wisniewski AB, Prendeville MT, Dobs AS. Handedness, functional cerebral hemispheric lateralization, and cognition in male-to-female transsexuals receiving cross-sex hormone treatment. Arch Sex Behav. 2005;34(2):167-72.

127. Slabbekoorn D, Van Goozen SH, Megens J, Gooren LJ, Cohen-Kettenis PT. Activating effects of cross-sex hormones on cognitive functioning: a study of short-term and long-term hormone effects in transsexuals. Psychoneuroendocrinology. 1999;24(4):423-47.

128. Van Goozen SHM, Cohen-Kettenis PT, Gooren LJG, Frijda NH, Van de Poll NE. Activating effects of androgens on cognitive performance: causal evidence in a group of female-to-male transsexuals. Neuropsychologia. 1994;32:1153-7.

129. Van Goozen SHM, Cohen-Kettenis PT, Gooren LJG, Frijda NH, Van de Poll NE. Gender differences in behaviour: activating effects of cross-gender hormones. Psychoneuroendocrinology. 1995;20:343-63.

130. Paap MCS, Haraldsen IR. Sex-based differences in answering strategy and the influence of cross-sex hormones. Psychiatry Res. 2010;175(3):266-70.

131. Haraldsen IR, Egeland T, Haug E, Finset A, Opjorsdsmoen S. Cross-sex hormone treatment does not change sex-sensitive cognitive performance in gender identity disorder patients. Psychiatry Res. 2005;137:161-74.

132. Miles $C$, Green $R$, Hines M. Estrogen effects on cognition, memory and mood in male-to-female transsexuals. Horm Behav. 2006;50:707-8.

133. Terada S, Matsumoto Y, Sato T, Okabe N, Kishimoto Y, Uchitomi Y. Suicidal ideation among patients with gender identity disorder. Psychiatry Res. 2011;190 (1):159-62.

134. Clements-Nolle K, Marx R, Katz M. Attempted suicide among transgender persons: the influence of gender-based discrimination and victimization. J Homosex. 2006;51(3):53-69.

135. Almeida J, Johnson RM, Corliss HL, Molnar BE, Azrael D. Emotional distress among LGBT youth: the influence of perceived discrimination based on sexual orientation. J Youth Adolesc. 2009;38(7):1001-14.

136. Grant JM, Mottet LA, Tanis J, Harrison J, Herman JL, Keisling M. Injustice at every turn: a report of the national transgender discrimination survey. National Center for Transgender Equality \& National Gay and Lesbian Task Force. Critical national survey describing the recent social context and status of transgender individuals in the US; 2011.

137. Haas AP, Eliason M, Mays VM, et al. Suicide and suicide risk in lesbian, gay, bisexual, and transgender populations: review and recommendations. J Homosex. 2011;58(1):10-51.

138. Maguen S, Shipherd JC. Suicide risk among transgender individuals. Psychol Sex. 2010;1(1):34-43.

139. Dhejne $C$, Lichtenstein $P$, Boman $M$, Johansson $A L$, Långström $N$, Landén $M$ Long-term follow-up of transsexual persons undergoing sex reassignment surgery: cohort study in Sweden. PLOS ONE. 2011;6(2):e16885.

140. Claes L, Bouman WP, Witcomb G, Thurston M, Fernandez-Aranda F, Arcelus J. Non-suicidal self-injury in trans people: associations with psychological symptoms, victimization, interpersonal functioning, and perceived social support. J Sex Med. 2015;12(1):168-79.

141. Hoshiai M, Matsumoto $Y$, Sato T, Ohnishi M, Okabe N, Kishimoto $Y$, et al. Psychiatric comorbidity among patients with gender identity disorder. Psychiatry Clin Neurosci. 2010;64(5):514-9.

142. Haraldsen IR, Dahl AA. Symptom profiles of gender dysphoric patients of transsexual type compared to patients with personality disorders and healthy adults. Acta Psychiatr Scand. 2000;102(4):276-81.

143. Bockting WO, Miner MH, Swinburne Romaine RE, Hamilton A, Coleman E. Stigma, mental health, and resilience in an online sample of the US transgender population. Am J Public Health. 2013;103(5):943-51.

144. Fisher AD, Castellini G, Ristori J, Casale H, Cassioli E, Sensi C, et al. Cross-sex hormone treatment and psychobiological changes in transsexual persons: twoyear follow-up data. J Clin Endocrinol Metab. 2016;101(11):4260-9.
145. Gorin-Lazard A, Baumstarck K, Boyer L, Maquigneau A, Penochet JC, Pringuey D, et al. Hormonal therapy is associated with better self-esteem, mood, and quality of life in transsexuals. J Nerv Ment Dis. 2013;201(11):996-1000.

146. van Kemenade JF, Cohen-Kettenis PT, Cohen L, Gooren LJ. Effects of the pure antiandrogen RU 23.903 (anandron) on sexuality, aggression, and mood in male-to-female transsexuals. Arch Sex Behav. 1989;18(3):217-28.

147. Leavitt F, Berger JC, Hoeppner JA, Northrop G. Presurgical adjustment in male transsexuals with and without hormonal treatment. J Nerv Ment Dis. 1980;168 (11):693-7.

148. Schulster M, Bernie AM, Ramasamy R. The role of estradiol in male reproductive function. Asian J Androl. 2016;18(3):435.

149. Epperson CN, Sammel MD, Bale TL, Kim DR, Conlin S, Scalice S, et al. Adverse childhood experiences and risk for first-episode major depression during the menopause transition. J Clin Psychiatry. 2017;78(3):e298-e307.

150. Nave G,Nadler A,Zava D,Camerer C, Single dose testosterone administration impairs cognitive reflection in men. Psychol Sci. 2017;28:1398-407.

151. Santoro N, Epperson CN, Mathews SB. Menopausal symptoms and their management. Endocrinol Metab Clin North Am. 2015;44(3):497-515.

152. Batrinos ML. Testosterone and aggressive behavior in man. Int J Endocrinol Metab. 2012;10(3):563.

153. van Honk J, Schutter DJ, Hermans EJ, Putman P, Tuiten A, Koppeschaar H. Testosterone shifts the balance between sensitivity for punishment and reward in healthy young women. Psychoneuroendocrinology. 2004;29(7):937-43.

154. Bishop KM, Wahlsten D. Sex differences in the human corpus callosum: myth or reality? Neurosci Biobehav Rev. 1997;21(5):581-601.

155. Weissman M, Bland R, Canino C, Faravelli C, Greenwald S, Hwu H, Joyce P, Karam E, Lee C, Lellouch J, Lépine J, Newman S, Rubio-Stipec M, Wells J, Wickramaratne $\mathrm{P}$, Wittchen $\mathrm{H}$, Yeh $\mathrm{E}$. Cross-national epidemiology of major depression and bipolar disorder. JAMA. 1996;276:293-9.

156. Weissman M, Bland R, Canino G, Greenwald S, Hwu H, Lee C, Newman S, OakleyBrowne $M$, Rubio-Stipec $M$, Wickramaratne $P$, et al. The cross national epidemiology of obsessive compulsive disorder: the cross national collaborative group. J Clin Psychiatry. 1994;55(Suppl.):5-10.

157. Blanton RE, Levitt JG, Peterson JR, Fadale D, Sporty ML, Lee M, et al. Gender differences in the left inferior frontal gyrus in normal children. Neuroimage. 2004;22:626-36.

158. DeLacoste-Utamsing C, Holloway RL. Sexual dimorphism in the human corpus callosum. Science. 1982;216(4553):1431-2.

159. Allen LS, Richey MF, Chai YM, Gorski RA. Sex differences in the corpus callosum of the living human being. J Neurosci. 1991;11(4):933-42.

160. Emory LE, Williams DH, Cole CM, Amparo EG, Meyer WJ. Anatomic variation of the corpus callosum in persons with gender dysphoria. Arch Sex Behav. 1991;20 (4):409-17.

161. Gater R, Tansella M, Korten A, Tiemans B, Mavreas V, Olatawura M. Sex differences in the prevalence and detection of depressive and anxiety disorders in general health care settings: report from the World Health Organization Collaborative Study on Psychological Problems in General Health Care. Arch Gen Psychiatry. 1998;55:405-13.

162. Giedd JN, Blumenthal J, Jeffries NO, Castellanos FX, Liu H, Zijdenbos A, Paus T, Evans AC, Rapoport JL. Brain development during childhood and adolescence: a longitudinal MRI study. Nat Neurosci. 1999;2:861-3.

163. Gómez-Gil E, Cañizares S, Torres A, de la Torre F, Halperin I, Salamero M. Androgen treatment effects on memory in female-to-male transsexuals. Psychoneuroendocrinology. 2009;34:110e7

164. Kessler R, McGonagle K, Zhao S, Nelson C, Hughes M, Eshleman S, Wittchen $H$, Kendler K. Lifetime and 12-month prevalence of DSM-III-R psychiatric disorders in the United States. Results from the National Comorbidity Survey. Arch Gen Psychiatry. 1994;51:8-19.

165. Kessler R, Sonnega A, Bromet E, Hughes M, Nelson C. Posttraumatic stress disorder in the National Comorbidity Survey. Arch Gen Psychiatry. 1995; 52:1048-60. 